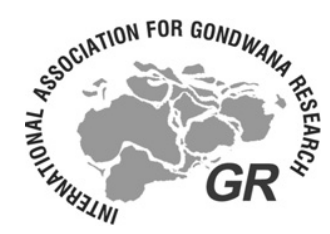

www.elsevier.com/locate/gr

\title{
Detrital zircons from upper Permian and lower Triassic Victoria Group sandstones, Shackleton Glacier region, Antarctica: Evidence for multiple sources along the Gondwana plate margin
}

\author{
D.H. Elliot ${ }^{\mathrm{a}, *}$, C.M. Fanning ${ }^{\mathrm{b}}$ \\ a Department of Geological Sciences and Byrd Polar Research Center, The Ohio State University, Columbus, OH 43210, USA \\ ${ }^{\mathrm{b}}$ Research School of Earth Sciences, Australian National University, Canberra, ACT 2000, Australia
}

Received 14 June 2006; accepted 1 May 2007

Available online 10 May 2007

\begin{abstract}
In the central Transantarctic Mountains region, siliciclastic strata of the Permian-Triassic Victoria Group (Beacon Supergroup), the Gondwana sequence in Antarctica, have both cratonic and magmatic arc provenance. Volcaniclastic strata occur in the upper part of the Permian Buckley Formation, whereas the Triassic Fremouw and Falla formations are more quartzose. Two sandstones in the upper Buckley and one near the base of the Fremouw, and immediately above the Permo-Triassic boundary, in the Shackleton Glacier region have yielded detrital zircon U/Pb SHRIMP age populations with clear evidence for a contemporaneous source. The stratigraphically lowest sample, about $70 \mathrm{~m}$ below the top of the Buckley Formation, is dominated by zoned igneous zircons of late Permian age. The sample from near the top of the Buckley Formation, about $40 \mathrm{~m}$ below the Permo-Triassic boundary, predominantly has zoned igneous zircons with the principal age peak again late Permian but with subordinate Devonian and Cambrian age peaks. The third sandstone from just above the Permo-Triassic boundary yielded zircons mainly of Neoproterozoic to Cambrian age. These results indicate a Permian-Triassic magmatic source existed during upper Buckley time, which is consistent with paleocurrent indicators of flow from the West Antarctic flank of the Permian-Triassic foreland basin, the inferred plate margin (Panthalassan) flank. The Devonian source is attributed to the Ford granodiorite suite in Marie Byrd Land, West Antarctica. Because Beacon strata cover the Cambrian Ross orogen and extend onto older basement, the Cambrian and Upper Proterozoic zircons are interpreted to be reworked from sandstones (Ordovician, Devonian, Early Permian in age) exposed in the Permian-Triassic Gondwanide orogen as a result of uplift of a fold and thrust belt by late Permian time.
\end{abstract}

(C) 2007 International Association for Gondwana Research. Published by Elsevier B.V. All rights reserved.

Keywords: Zircon; geochronology; Beacon; Antarctica; Permian-Triassic

\section{Introduction}

The siliciclastic fill of a sedimentary basin reflects source region lithologies, which also may indicate the tectonic framework. Epeirogenic downwarping of broad regions will commonly lead to relatively mature quartz-rich sandstones, whereas, for instance, rift basins will reflect the geology of the proximal rift shoulders. In the former case, sandstone and heavy mineral petrology yields only relatively sparse data, whereas in the latter case, it can provide much provenance information. In most cases, the sand-sized grains commonly reflect mixed

\footnotetext{
* Corresponding author. Tel.: +1 614292 5076; fax: +1 6142927688 .
}

E-mail address: elliot.1@osu.edu (D.H. Elliot). sources. Detrital zircons yield complementary data, which may include: populations of grains with different ages; evidence for recycling of grains; evidence for igneous or metamorphic origin; and isotopic data that allow identification of crustal source terrains (e.g. Ross et al., 1992; Geslin et al., 1999; Hallsworth et al., 2000; Adams et al., 2002; Ross and Villeneuve, 2003; Goodge et al., 2004a,b; Link et al., 2005).

The sandstone petrology of the Victoria Group (the Permian-Triassic part of the Beacon Supergroup) as a whole has been reported (Vavra et aly., 1981; Barrett et al., 1986; Collinson et al., 1994) to include quartzose sandstones of probable cratonic origin, and volcaniclastic sandstones derived from a magmatic arc. This paper reports a preliminary study of detrital zircons in sandstones from the upper Permian and 
lowest Triassic part of the Victoria Group in the Shackleton Glacier area in the central Transantarctic Mountains (CTM). It is the first step in differentiating source terrains for the Victoria Group, and infers when sources were active and the possible timing of tectonic events in the Gondwanide orogenic belt.

\section{Regional geology}

Devonian to Triassic Beacon Supergroup strata crop out in the Transantarctic Mountains (TAM), and overlie a preDevonian basement of igneous and metamorphic rocks (Fig. 1). The basement is formed mainly of Neoproterozoic to Cambrian sedimentary and volcanic strata, which were deformed, metamorphosed and intruded by granitoid batholiths in the Cambro-Ordovician Ross Orogeny (Stump, 1995). A regional erosion surface was cut across the Ross Orogen in preDevonian time. On this surface were deposited quartz-rich sandstones and minor finer grained beds of the Devonian Taylor

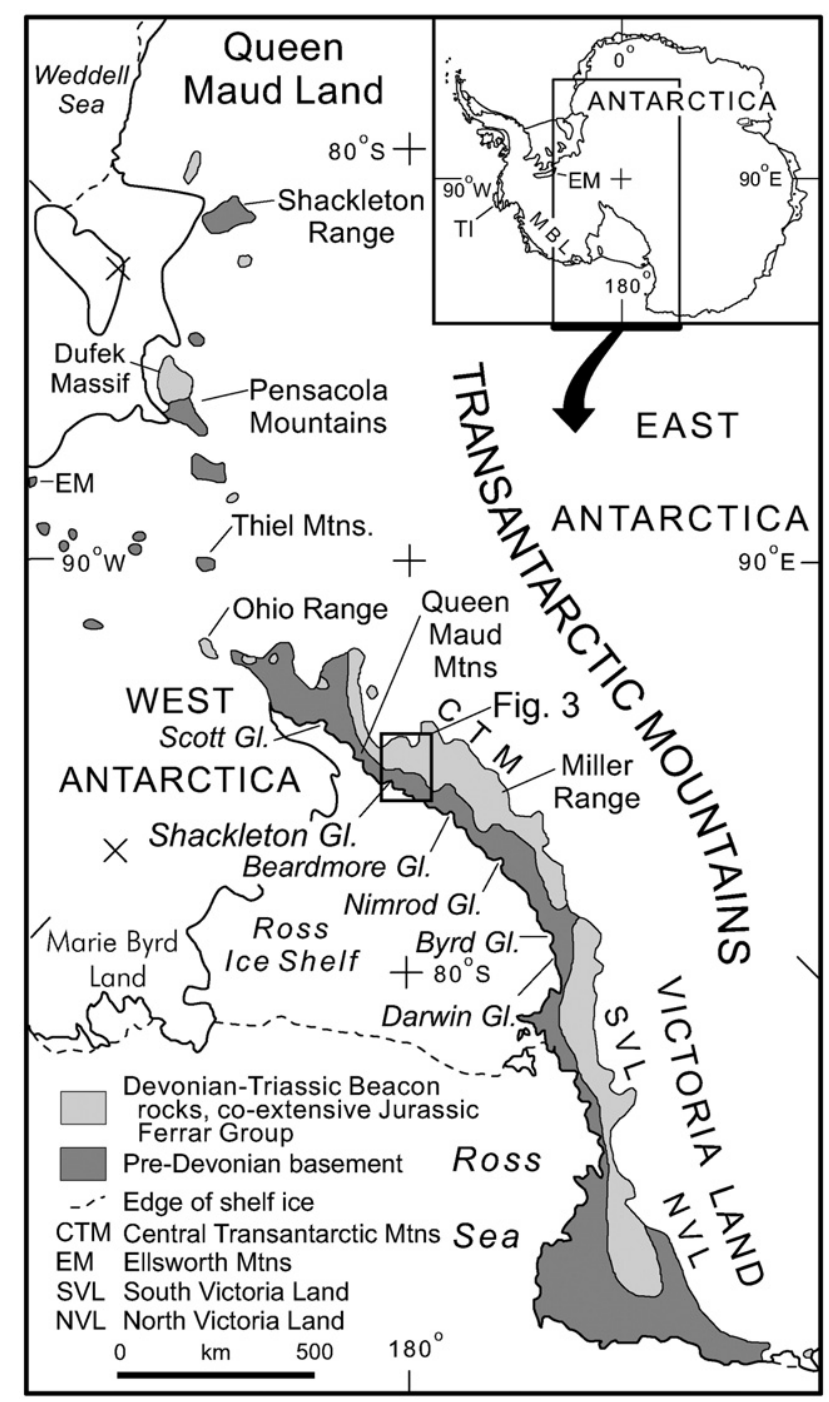

Fig. 1. Location map for Devonian to Triassic Beacon Supergroup strata in the Transantarctic Mountains, Antarctica. EM: Ellsworth Mountains; MBL: Marie Byrd Land; TI: Thurston Island.

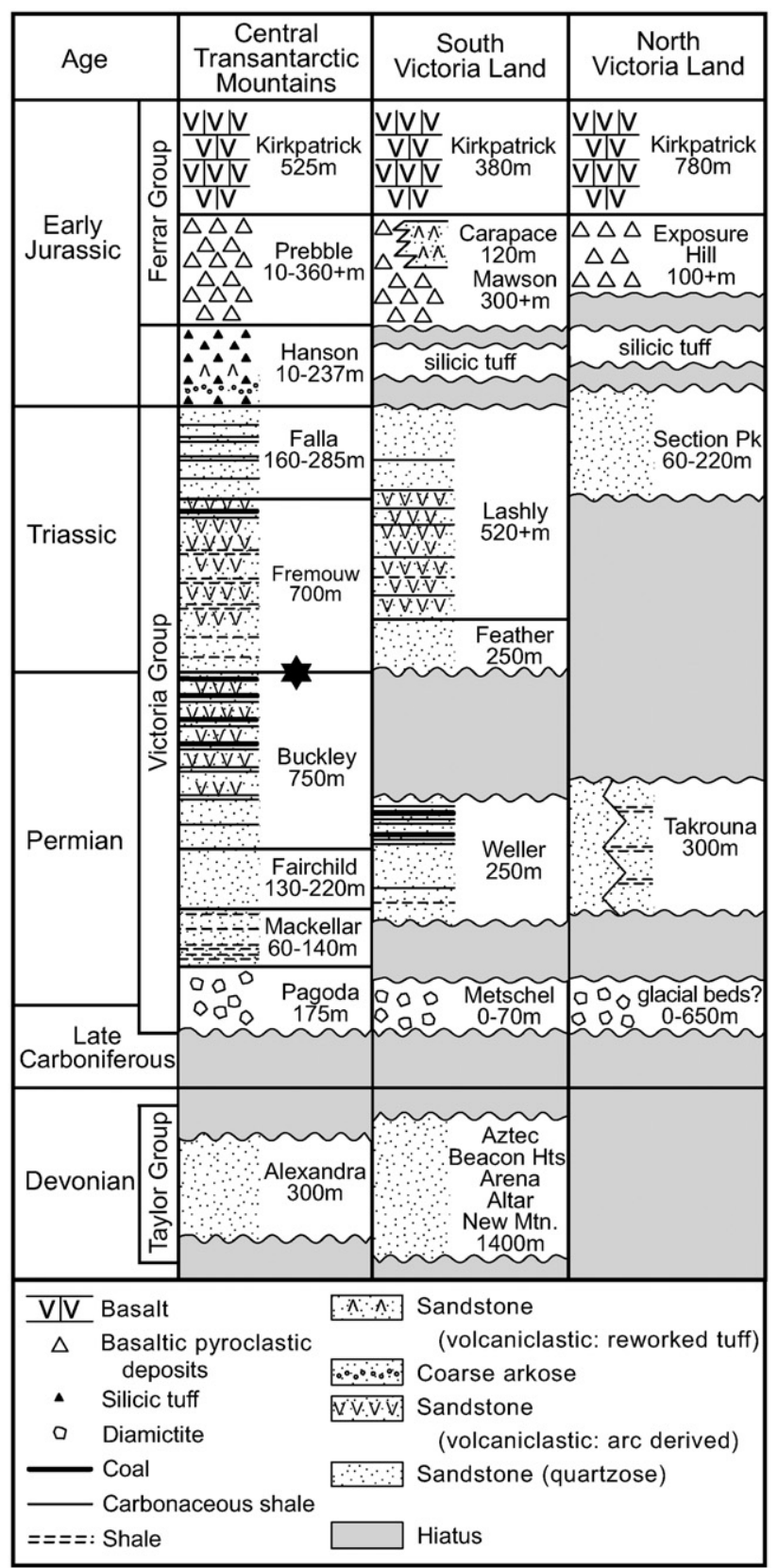

Fig. 2. Beacon Supergroup stratigraphy (Taylor and Victoria Groups) in the Transantarctic Mountains. Star on the central Transantarctic Mountains column marks the location of analyzed samples. See Collinson et al. (1994) for details of stratigraphy.

Group (Fig. 2). A second erosion surface was cut prior to deposition of the Permian-Triassic Victoria Group, the Antarctic Gondwana sequence. The Beacon strata were overlain by extrusive rocks of the Ferrar large igneous province and intruded by co-magmatic sills and dikes.

The East Antarctic craton under the polar ice sheet is inferred to include pan-African age belts, Grenville age provinces, Proterozoic domains, and Archean cratons (Fitzsimons, 2000, 2003); a large extension of the Archean-Paleoproterozoic Mawson Continent is inferred to dominate the ice-covered hinterland to the TAM and extend to adjacent areas in Australia (Fanning et al., 1996). Archean gneisses, initially emplaced at 
ca. 2.98 Ma, metamorphosed during a high-grade event at ca. 2.96 Ma and intruded by $2.5 \mathrm{Ga}$ granites, form the oldest exposed basement rocks (Goodge and Fanning, 1999). These Archaean gneisses were reworked by Paleoproterozoic metamorphism and syn-tectonic magmatism at $1.7 \mathrm{Ga}$ and again during the Ross Orogeny (Goodge et al., 2001; Goodge and Fanning, 2002). In outcrop they are restricted to the Miller Range region in CTM (Fig. 1).

Neoproterozoic to Ordovician strata are widespread in the TAM (Goodge et al., 2004a,b). Neoproterozoic to Lower Cambrian rift-related siliciclastic strata are confined to the Pensacola Mountains (Hannah Ridge Formation) and CTM (Beardmore Group). Cambrian to lower Ordovician siliciclastic strata and associated carbonate shelf deposits are widespread between the Byrd Glacier and the Pensacola Mountains region (Byrd Group and the correlative Patuxent Formation). Cambrian silicic volcanic rocks are common in the Queen Maud Mountains and Thiel Mountains (Pankhurst et al., 1988; Wareham et al., 2001). Granitoids were emplaced in the late Cambrian during the Ross Orogeny, both pre-deformation, the main phase of which was over by about $500 \mathrm{Ma}$, and locally post-deformation (Borg et al., 1990; summarized in Rowell et al., 2001). North of Byrd Glacier, in south and north Victoria Land (SVL, NVL), the basement is dominated by medium to high-grade metamorphic rocks, which were intruded by Ross age granitoids that again show pre-and post-tectonic phases. In SVL, the metamorphic rocks (Koettlitz Group) are probably correlative with the Neoproterozoic metasediments in CTM (Goodge et al., 2004a). Detrital zircons from the Upper Proterozoic to Lower Ordovician strata show a wide range of provenance ages, ranging back to the Archean but dominated by Cambrian and both Neo-and Mesoproterozoic ages (Goodge et al., 2004a,b).

West Antarctica is part of the dismembered Gondwana plate margin (Dalziel and Elliot, 1982), now consisting of continental blocks and severely stretched continental crust with thicknesses of 20-25 km (Winberry and Anandakrishnan, 2004). Much of West Antarctica is ice covered but outcrops through Marie Byrd Land (MBL) and Thurston Island (Fig. 1) indicate the presence of Paleozoic to Early Mesozoic (Triassic) magmatic arcs which are recorded principally as granitoids (Pankhurst et al., 1993, 1998; Mukasa and Dalziel, 2000). On the basis of geochronologic and geochemical data, an inboard Ross Province with Cambrian metagreywackes and gneisses has been defined in western MBL and an outboard Amundsen Province in eastern MBL that lacks Cambrian age rocks (Pankhurst et al., 1998). The Ross Province includes Cambrian orthogneisses, with a not well-constrained age of about $505 \mathrm{Ma}$, and Swanson Formation greywackes with metamorphic ages of 440-420 Ma (Adams et al., 1995); however, the principal rocks in the Ross Province are intrusive and belong to the Ford Granodiorite, which forms a well-defined set of Devonian plutons with an emplacement age of $375 \pm 5 \mathrm{Ma}$ (Pankhurst et al., 1998). This magmatic belt extends into NVL (Henjes-Kuntz and Kreuzer, 2003, and references therein), and into New Zealand (summarized in Wandres and Bradshaw, 2005); orthogneisses of that age are known from the Antarctic Peninsula (Millar et al., 2002). Carboniferous granitic bodies with ages about $340 \mathrm{Ma}$ are also present in MBL. Orthogneisses on Thurston Island and a leucogranite on the Antarctic Peninsula have similar ages. The Amundsen Province includes Ordovician to Silurian (450-420 Ma) granitoids, but it is characterized principally by a Permian-Triassic magmatic arc which crops out in central and eastern MBL and Thurston Island. Millar et al. (2002) report sparse granite and orthogneiss of that age in the Antarctic Peninsula.

Denudation following the Ross Orogeny led to the formation of the Kukri Erosion Surface throughout the TAM (Isbell, 1999). The Taylor Group was deposited on this surface and now crops out principally in SVL where the sequence, up to $1400 \mathrm{~m}$ thick, consists of quartzose sandstones overlain by a small interval of finer grained beds from which Devonian fossils have been recovered (Bradshaw and Webers, 1988; Bradshaw, 1991). Quartz-rich sandstones of presumed Devonian age continue south to a paleotopographic high in the Nimrod Glacier region and are present again farther south (north of the Beardmore Glacier) where thicknesses exceed $300 \mathrm{~m}$ (Isbell, 1999). An uncertain small thickness occurs at Mt. Hermanson between the Beardmore and Ramsey Glaciers (Fig. 3), and about $60 \mathrm{~m}$ is present at Sullivan Ridge in the Ramsey Glacier region (Coates, 1985). A paleotopographic high separates these siliciclastic sequences from the marine Devonian beds in the Ohio Range (Bradshaw and Webers, 1988). The Kukri surface has

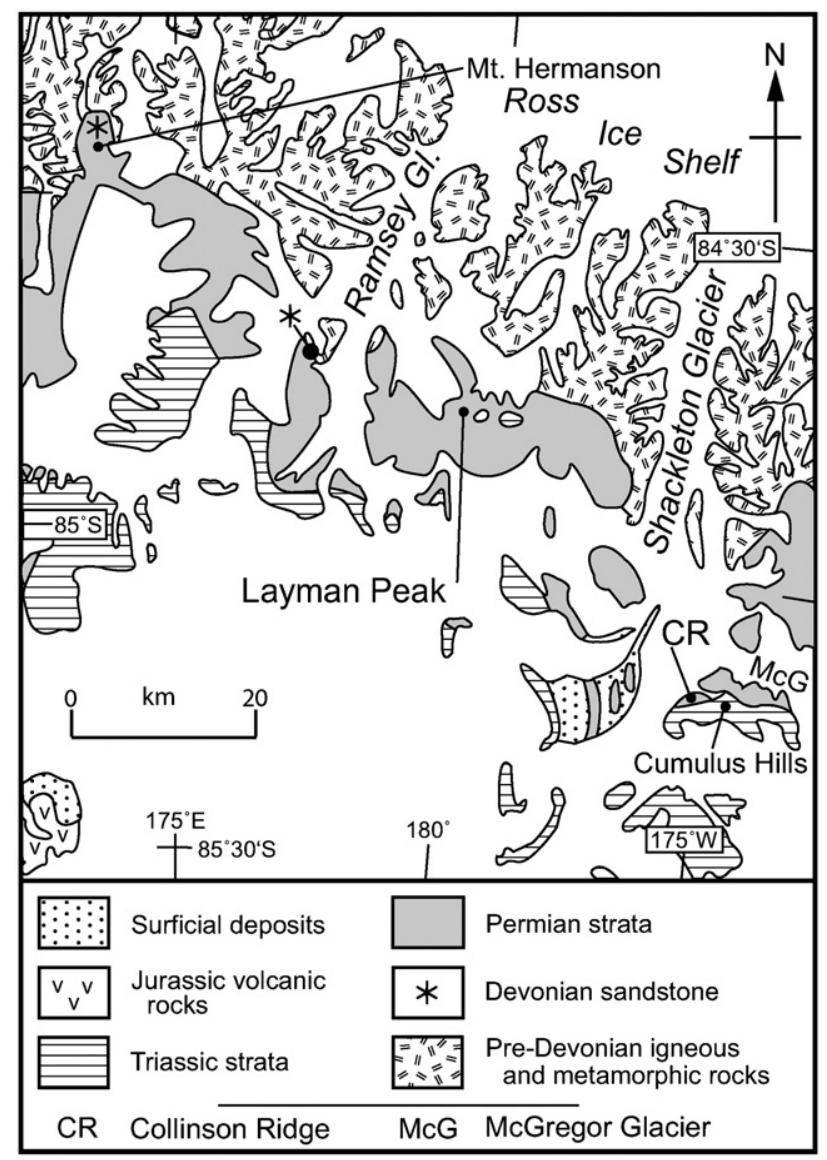

Fig. 3. Locality and simplified geologic map of the Shackleton Glacier region. (Note: orientation reversed from Fig. 1). 
considerable relief, locally as much as $700 \mathrm{~m}$ south of Byrd Glacier (Anderson, 1979).

The younger, pre-glacial (Permian) Maya Erosion Surface was superimposed on the Kukri surface and Devonian strata, and also has considerable relief (Isbell, 1999). Uncertain amounts of Devonian strata were probably removed before deposition of the Permo-Triassic Victoria Group (Collinson et al., 1994) on this surface. Three depositional basins have been recognized (see Fig. 2 for stratigraphy): intra-cratonic basins in NVL and SVL, and to the south of the Byrd Glacier a cratonic basin that evolved in middle Permian time into a foreland basin.

In this basin south of Byrd Glacier, the Permian glacial beds (Fig. 2) are overlain by post-glacial shales that transition through quartzose sandstones upwards into fluvial coal measures of Middle and Late Permian age. These are succeeded by Triassic flood plain sediments largely devoid of coal. Sedimentation ended in late Triassic time, and during the Early Jurassic a silicic volcaniclastic sequence (Hanson Formation; Elliot, 1996) was deposited in a rift setting that had developed on the earlier foreland basin. In the late Early Jurassic, Ferrar tholeiitic magmas were erupted as pyroclastic rocks and lava flows, and intruded as dolerite sills and dikes (Elliot and Fleming, in press). Given the thickness of the exposed strata, the foreland basin must have extended onto the craton and also toward the active margin.

Paleocurrent analysis of the foreland basin strata in the Shackleton Glacier region (Vavra et al., 1981; Isbell, 1991) shows derivation of detrital material from quartzose sources during the early Permian and flow generally towards the southeast along the mountain range. This pattern was replaced by magmatic arc volcaniclastic sources on the West Antarctic flank during middle and Late Permian deposition of the coal measures. Volcaniclastic sources were relatively less important in the Triassic, but paleocurrents still indicate derivation from the magmatic arc flank of the basin. Ash beds and shard-bearing sedimentary rocks show that contemporaneous volcanism continued at least into Middle Triassic time. The advent of volcaniclastic sources was accompanied by a gradual reversal in major trunk drainage and by the late Triassic the foreland basin was linked to the intracratonic basins in SVL and NVL, with paleocurrent flow towards Australia. The major paleocurrent reversal of the trunk drainage is attributed to the development of a fold and thrust belt, which is documented in the Pensacola and Ellsworth Mountains (Collinson et al., 1994).

\section{Beacon strata in the Shackleton Glacier region}

Taylor Group quartzose sandstones are represented by a single outcrop in the Ramsey Glacier region (Fig. 3) (Coates, 1985). In most places Victoria Group strata rest nonconformably on Neoproterozoic metasedimentary and meta-igneous rocks or on Ross granitoids (Collinson et al., 1994; Isbell, 1999). The lower Permian formations (Pagoda, Mackellar, and Fairchild) crop out in the foothills of the range, and between the Ramsey and Shackleton Glaciers are overlain by the Permian coal measures (Buckley Formation), which locally are capped by Lower Triassic beds of the Fremouw Formation. In the McGregor Glacier region the upper part of the Buckley
Formation and the Fremouw Formation are well exposed in the northern Cumulus Hills. Both the Fremouw and Falla Formations are well exposed farther south adjacent to the Shackleton Glacier (Collinson and Elliot, 1984). Paleocurrent measurements indicate derivation of the clastic debris in both the upper Buckley and lower Fremouw Formations from West Antarctica (Vavra et al., 1981; Isbell et al., 1997).

A simplified and composite stratigraphic column (Fig. 4) shows the principal sandstones in the upper Buckley and lower Fremouw flood plain sequences. The lower part was measured at Layman Peak and the upper part at Collinson Ridge, Cumulus Hills (see also Collinson et al., 2006). Samples analyzed come from $70 \mathrm{~m}$ (sample 35-2) and $3 \mathrm{~m}$ (sample 36-1) below the Buckley/Fremouw contact, and from $45 \mathrm{~m}$ (sample 36-4) in the Fremouw Formation, in the channel sandstone containing the first vertebrates. Sample $36-1$ is $2 \mathrm{~m}$ above a $100+\mathrm{m}$ thick dolerite sill,

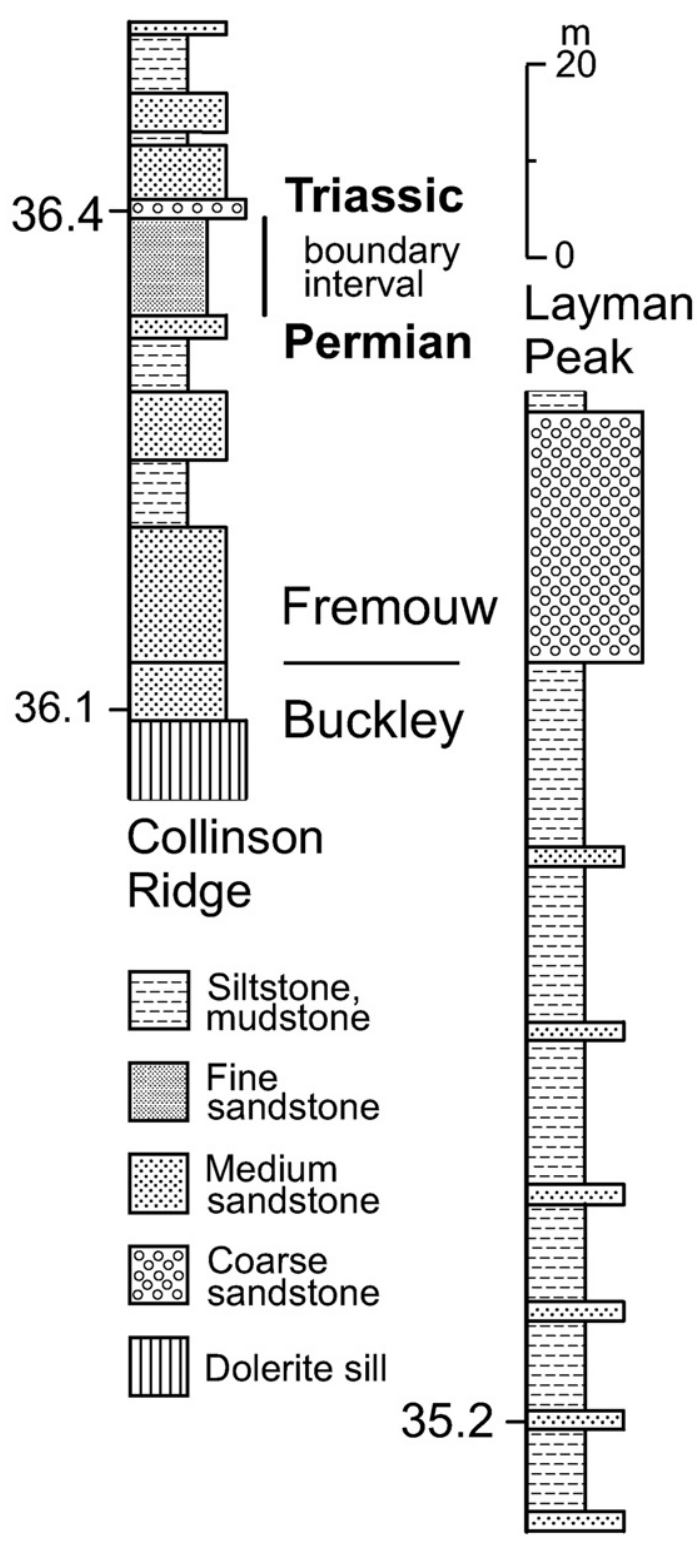

Fig. 4. Simplified stratigraphic columns for the upper Buckley Formation and lower Fremouw Formation in the Shackleton Glacier area. (see Fig. 3 for locations). 
Table 1

Summary of SHRIMP U-Pb zircon results for sample 96-35-2

\begin{tabular}{|c|c|c|c|c|c|c|c|c|c|c|c|c|c|c|c|c|c|c|c|c|c|c|}
\hline \multirow{2}{*}{$\begin{array}{l}\text { Grain } \\
\text { spot }\end{array}$} & \multirow{2}{*}{$\begin{array}{l}\mathrm{U} \\
\text { (ppm) }\end{array}$} & \multirow{2}{*}{$\begin{array}{l}\text { Th } \\
\text { (ppm) }\end{array}$} & \multirow[t]{2}{*}{$\mathrm{Th} / \mathrm{U}$} & \multirow{2}{*}{$\begin{array}{l}\mathrm{Pb}^{*} \\
\text { (ppm) }\end{array}$} & \multirow{2}{*}{$\begin{array}{l}{ }^{204} \mathrm{~Pb} / \\
{ }^{206} \mathrm{~Pb}\end{array}$} & \multirow{2}{*}{$\begin{array}{l}\mathrm{f}_{206} \\
\%\end{array}$} & \multicolumn{4}{|c|}{ Total ratios } & \multicolumn{6}{|c|}{ Radiogenic ratios } & \multirow[t]{2}{*}{$\rho$} & \multicolumn{4}{|c|}{ Age (Ma) } & \multirow{2}{*}{$\begin{array}{l}\% \\
\text { disc }\end{array}$} \\
\hline & & & & & & & ${ }^{238} \mathrm{U} /$ & \pm & $\begin{array}{l}{ }^{207} \mathrm{~Pb} / \\
{ }^{206} \mathrm{~Pb}\end{array}$ & \pm & $\begin{array}{l}{ }^{206} \mathrm{~Pb} / \\
{ }^{238} \mathrm{U}\end{array}$ & \pm & $\begin{array}{l}{ }^{207} \mathrm{~Pb} / \\
{ }^{235} \mathrm{U}\end{array}$ & \pm & $\begin{array}{l}{ }^{207} \mathrm{~Pb} / \\
{ }^{206} \mathrm{~Pb}\end{array}$ & \pm & & $\begin{array}{l}{ }^{206} \mathrm{~Pb} / \\
{ }^{238} \mathrm{U}\end{array}$ & \pm & $\begin{array}{l}{ }^{207} \mathrm{~Pb} / \\
{ }^{206} \mathrm{~Pb}\end{array}$ & \pm & \\
\hline 1.1 & 329 & 224 & 0.68 & 11.4 & - & 0.13 & 24.739 & 0.351 & 0.0524 & 0.0012 & 0.0404 & 0.0006 & & & & & & 255.1 & 3.6 & & & \\
\hline 2.1 & 372 & 170 & 0.46 & 14.0 & 0.000130 & $<0.01$ & 22.882 & 0.316 & 0.0506 & 0.0010 & 0.0438 & 0.0006 & & & & & & 276.1 & 3.8 & & & \\
\hline 3.1 & 494 & 215 & 0.44 & 18.3 & 0.000137 & 0.08 & 23.137 & 0.300 & 0.0524 & 0.0009 & 0.0432 & 0.0006 & & & & & & 272.5 & 3.5 & & & \\
\hline 4.1 & 291 & 125 & 0.43 & 9.9 & 0.000388 & 0.64 & 25.350 & 0.389 & 0.0563 & 0.0014 & 0.0392 & 0.0006 & & & & & & 247.9 & 3.8 & & & \\
\hline 5.1 & 209 & 202 & 0.96 & 7.7 & 0.000022 & 0.09 & 23.289 & 0.369 & 0.0524 & 0.0015 & 0.0429 & 0.0007 & & & & & & 270.8 & 4.3 & & & \\
\hline 6.1 & 158 & 159 & 1.00 & 5.7 & 0.000374 & 0.19 & 23.642 & 0.406 & 0.0531 & 0.0016 & 0.0422 & 0.0007 & & & & & & 266.6 & 4.5 & & & \\
\hline 7.1 & 299 & 145 & 0.48 & 10.9 & - & 0.14 & 23.450 & 0.340 & 0.0527 & 0.0012 & 0.0426 & 0.0006 & & & & & & 268.8 & 3.9 & & & \\
\hline 8.1 & 413 & 215 & 0.52 & 15.3 & 0.000078 & 0.24 & 23.195 & 0.321 & 0.0536 & 0.0011 & 0.0430 & 0.0006 & & & & & & 271.5 & 3.7 & & & \\
\hline 9.1 & 69 & 47 & 0.67 & 9.9 & 0.000094 & 0.16 & 6.003 & 0.109 & 0.0808 & 0.0015 & 0.1663 & 0.0030 & 1.824 & 0.064 & 0.0795 & 0.0024 & 0.521 & 992 & 17 & 1185 & 59 & 16 \\
\hline 10.1 & 244 & 138 & 0.56 & 8.4 & 0.000533 & $<0.01$ & 25.124 & 0.382 & 0.0509 & 0.0013 & 0.0398 & 0.0006 & & & & & & 251.7 & 3.8 & & & \\
\hline 11.1 & 466 & 190 & 0.41 & 17.5 & - & 0.01 & 22.839 & 0.304 & 0.0518 & 0.0010 & 0.0438 & 0.0006 & & & & & & 276.2 & 3.6 & & & \\
\hline 12.1 & 169 & 110 & 0.65 & 5.8 & 0.000832 & 0.34 & 25.086 & 0.429 & 0.0539 & 0.0016 & 0.0397 & 0.0007 & & & & & & 251.2 & 4.3 & & & \\
\hline 13.1 & 592 & 719 & 1.21 & 21.9 & - & 0.11 & 23.280 & 0.333 & 0.0526 & 0.0009 & 0.0429 & 0.0006 & & & & & & 270.8 & 3.8 & & & \\
\hline 14.1 & 116 & 48 & 0.41 & 4.6 & - & $<0.01$ & 21.609 & 0.437 & 0.0509 & 0.0019 & 0.0463 & 0.0010 & & & & & & 292.1 & 5.9 & & & \\
\hline 15.1 & 268 & 113 & 0.42 & 9.0 & - & 0.23 & 25.667 & 0.453 & 0.0529 & 0.0014 & 0.0389 & 0.0007 & & & & & & 245.8 & 4.3 & & & \\
\hline 16.1 & 471 & 303 & 0.64 & 15.9 & - & 0.18 & 25.465 & 0.336 & 0.0526 & 0.0010 & 0.0392 & 0.0005 & & & & & & 247.9 & 3.2 & & & \\
\hline 17.1 & 299 & 126 & 0.42 & 10.5 & 0.000400 & 1.40 & 24.494 & 0.359 & 0.0626 & 0.0022 & 0.0403 & 0.0006 & & & & & & 254.4 & 3.8 & & & \\
\hline 18.1 & 140 & 117 & 0.83 & 4.9 & - & 0.02 & 24.362 & 0.427 & 0.0516 & 0.0017 & 0.0410 & 0.0007 & & & & & & 259.3 & 4.5 & & & \\
\hline 19.1 & 111 & 99 & 0.89 & 4.2 & - & 0.47 & 22.939 & 0.451 & 0.0555 & 0.0020 & 0.0434 & 0.0009 & & & & & & 273.8 & 5.4 & & & \\
\hline 20.1 & 238 & 135 & 0.57 & 23.0 & 0.000498 & 0.59 & 8.889 & 0.124 & 0.0671 & 0.0010 & 0.1118 & 0.0016 & & & & & & 683.4 & 9.3 & & & \\
\hline 21.1 & 291 & 161 & 0.55 & 9.7 & 0.000070 & 0.41 & 25.798 & 0.410 & 0.0544 & 0.0025 & 0.0386 & 0.0006 & & & & & & 244.2 & 3.9 & & & \\
\hline 22.1 & 630 & 612 & 0.97 & 22.2 & 0.000159 & $<0.01$ & 24.340 & 0.299 & 0.0511 & 0.0008 & 0.0411 & 0.0005 & & & & & & 259.7 & 3.2 & & & \\
\hline 23.1 & 119 & 91 & 0.77 & 4.2 & 0.000382 & 0.11 & 24.049 & 0.469 & 0.0523 & 0.0019 & 0.0415 & 0.0008 & & & & & & 262.3 & 5.1 & & & \\
\hline 24.1 & 312 & 197 & 0.63 & 11.6 & - & $<0.01$ & 23.099 & 0.335 & 0.0512 & 0.0012 & 0.0433 & 0.0006 & & & & & & 273.4 & 3.9 & & & \\
\hline 25.1 & 165 & 106 & 0.64 & 5.9 & 0.000167 & 0.37 & 23.880 & 0.407 & 0.0545 & 0.0019 & 0.0417 & 0.0007 & & & & & & 263.5 & 4.5 & & & \\
\hline 26.1 & 176 & 200 & 1.13 & 6.3 & 0.000503 & 0.30 & 23.822 & 0.402 & 0.0540 & 0.0016 & 0.0419 & 0.0007 & & & & & & 264.3 & 4.4 & & & \\
\hline 27.1 & 243 & 122 & 0.50 & 8.7 & - & 0.36 & 23.861 & 0.359 & 0.0544 & 0.0013 & 0.0418 & 0.0006 & & & & & & 263.7 & 3.9 & & & \\
\hline 28.1 & 132 & 154 & 1.17 & 4.8 & - & 1.23 & 23.562 & 0.440 & 0.0614 & 0.0020 & 0.0419 & 0.0008 & & & & & & 264.7 & 4.9 & & & \\
\hline 29.1 & 231 & 136 & 0.59 & 8.4 & - & $<0.01$ & 23.700 & 0.362 & 0.0514 & 0.0013 & 0.0422 & 0.0007 & & & & & & 266.5 & 4.0 & & & \\
\hline 30.1 & 330 & 180 & 0.54 & 10.6 & 0.000176 & 0.44 & 26.644 & 0.396 & 0.0544 & 0.0013 & 0.0374 & 0.0006 & & & & & & 236.5 & 3.5 & & & \\
\hline 31.1 & 469 & 294 & 0.63 & 17.3 & 0.000056 & 0.15 & 23.331 & 0.299 & 0.0528 & 0.0009 & 0.0428 & 0.0006 & & & & & & 270.2 & 3.4 & & & \\
\hline 32.1 & 289 & 204 & 0.71 & 10.3 & 0.000054 & $<0.01$ & 24.062 & 0.344 & 0.0506 & 0.0016 & 0.0416 & 0.0006 & & & & & & 262.8 & 3.7 & & & \\
\hline 33.1 & 161 & 102 & 0.63 & 5.5 & - & 0.32 & 25.135 & 0.437 & 0.0538 & 0.0022 & 0.0397 & 0.0007 & & & & & & 250.7 & 4.4 & & & \\
\hline 34.1 & 144 & 119 & 0.83 & 5.2 & 0.002122 & 3.73 & 23.903 & 0.431 & 0.0812 & 0.0031 & 0.0403 & 0.0008 & & & & & & 254.5 & 4.8 & & & \\
\hline 35.1 & 338 & 221 & 0.66 & 11.7 & 0.000046 & 0.36 & 24.705 & 0.347 & 0.0542 & 0.0012 & 0.0403 & 0.0006 & & & & & & 254.9 & 3.6 & & & \\
\hline 36.1 & 929 & 455 & 0.49 & 31.4 & 0.000388 & 0.48 & 25.453 & 0.297 & 0.0550 & 0.0007 & 0.0391 & 0.0005 & & & & & & 247.2 & 2.9 & & & \\
\hline 37.1 & 131 & 103 & 0.79 & 5.2 & 0.005904 & 8.38 & 21.590 & 0.421 & 0.1188 & 0.0228 & 0.0424 & 0.0016 & & & & & & 267.9 & 10.1 & & & \\
\hline 38.1 & 172 & 182 & 1.06 & 5.5 & 0.001488 & 1.62 & 26.840 & 0.504 & 0.0637 & 0.0022 & 0.0367 & 0.0007 & & & & & & 232.1 & 4.4 & & & \\
\hline 39.1 & 379 & 166 & 0.44 & 13.9 & - & $<0.01$ & 23.422 & 0.311 & 0.0516 & 0.0010 & 0.0427 & 0.0006 & & & & & & 269.5 & 3.5 & & & \\
\hline 40.1 & 160 & 95 & 0.60 & 5.2 & 0.000260 & 0.46 & 26.307 & 0.465 & 0.0546 & 0.0017 & 0.0378 & 0.0007 & & & & & & 239.4 & 4.2 & & & \\
\hline 41.1 & 230 & 240 & 1.04 & 8.6 & 0.007169 & 14.46 & 22.891 & 0.356 & 0.1667 & 0.0039 & 0.0374 & 0.0010 & & & & & & 236.5 & 6.3 & & & \\
\hline 42.1 & 422 & 213 & 0.51 & 14.4 & 0.000478 & 0.31 & 25.142 & 0.331 & 0.0537 & 0.0014 & 0.0396 & 0.0005 & & & & & & 250.7 & 3.3 & & & \\
\hline 43.1 & 287 & 257 & 0.90 & 30.9 & 0.008686 & 39.55 & 7.975 & 0.595 & 0.3780 & 0.0858 & 0.0758 & 0.0159 & & & & & & 471.0 & 95.0 & & & \\
\hline 44.1 & 172 & 147 & 0.86 & 6.3 & 0.000547 & 0.06 & 23.569 & 0.391 & 0.0521 & 0.0015 & 0.0424 & 0.0007 & & & & & & 267.7 & 4.4 & & & \\
\hline
\end{tabular}


Table 1 (continued)

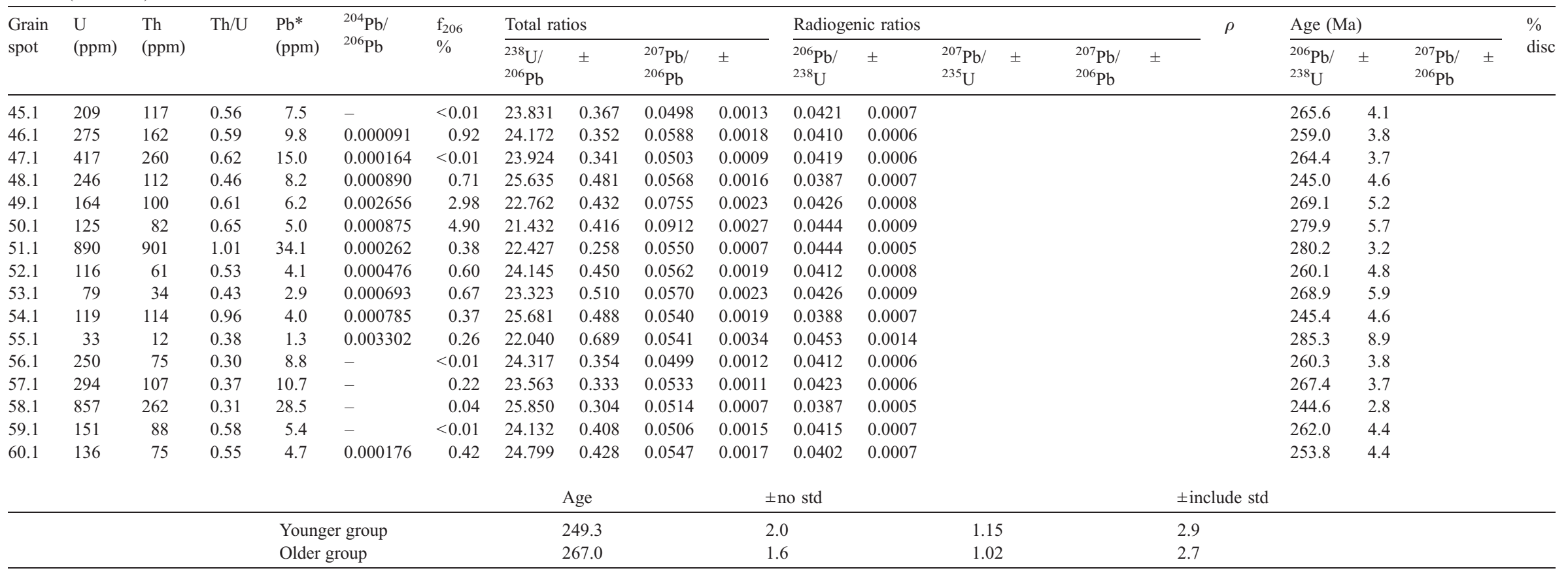

Notes:

1. Uncertainties given at the one $\sigma$ level.

2. Error in FC1 Reference zircon calibration was $0.82 \%$ for the analytical session. (not included in above errors but required when comparing ${ }^{206} \mathrm{~Pb} /{ }^{238} \mathrm{U}$ data from different mounts).

3. $\mathrm{f}_{206} \%$ denotes the percentage of ${ }^{206} \mathrm{~Pb}$ that is common $\mathrm{Pb}$.

4. For areas older than $\sim 800 \mathrm{Ma}$ correction for common $\mathrm{Pb}$ made using the measured ${ }^{204} \mathrm{~Pb} /{ }^{206} \mathrm{~Pb}$ ratio.

5. For areas younger than $\sim 800 \mathrm{Ma}$ correction for common $\mathrm{Pb}$ made using the measured ${ }^{238} \mathrm{U} /{ }^{206} \mathrm{~Pb}$ and ${ }^{207} \mathrm{~Pb} /{ }^{206} \mathrm{~Pb}$ ratios following Tera and Wasserburg (1972) as outlined in Williams (1998).

6. For $\%$ disc, $0 \%$ denotes a concordant analysis.

$\mathrm{Pb} *$ indicates radiogenic $\mathrm{Pb}$. 
Table 2

Summary of SHRIMP U-Pb zircon results for sample 96-36-1

\begin{tabular}{|c|c|c|c|c|c|c|c|c|c|c|c|c|c|c|c|c|c|c|c|c|c|}
\hline \multirow{2}{*}{$\begin{array}{l}\text { Grain } \\
\text { spot }\end{array}$} & \multirow{2}{*}{$\begin{array}{l}\mathrm{U} \\
(\mathrm{ppm})\end{array}$} & \multirow{2}{*}{$\begin{array}{l}\text { Th } \\
\text { (ppm) }\end{array}$} & \multirow[t]{2}{*}{$\mathrm{Th} / \mathrm{U}$} & \multirow{2}{*}{$\begin{array}{l}\mathrm{Pb}^{*} \\
(\mathrm{ppm})\end{array}$} & \multirow{2}{*}{$\begin{array}{l}{ }^{204} \mathrm{~Pb} / \\
{ }^{206} \mathrm{~Pb}\end{array}$} & \multirow{2}{*}{$\begin{array}{l}f_{206} \\
\%\end{array}$} & \multicolumn{4}{|c|}{ Total ratios } & \multicolumn{5}{|c|}{ Radiogenic ratios } & \multirow[t]{2}{*}{$\rho$} & \multicolumn{4}{|c|}{ Age (Ma) } & \multirow{2}{*}{$\begin{array}{l}\% \\
\text { disc }\end{array}$} \\
\hline & & & & & & & $\begin{array}{l}{ }^{238} \mathrm{U} / \\
{ }^{206} \mathrm{~Pb}\end{array}$ & \pm & $\begin{array}{l}{ }^{207} \mathrm{~Pb} / \\
{ }^{206} \mathrm{~Pb}\end{array}$ & \pm & $\begin{array}{l}{ }^{206} \mathrm{~Pb} / \\
{ }^{238} \mathrm{U}\end{array}$ & \pm & $\begin{array}{l}{ }^{207} \mathrm{~Pb} / \pm \\
{ }^{235} \mathrm{U}\end{array}$ & $\begin{array}{l}{ }^{207} \mathrm{~Pb} / \\
{ }^{206} \mathrm{~Pb}\end{array}$ & \pm & & $\begin{array}{l}{ }^{206} \mathrm{~Pb} / \\
{ }^{238} \mathrm{U}\end{array}$ & \pm & $\begin{array}{l}{ }^{207} \mathrm{~Pb} / \\
{ }^{206} \mathrm{~Pb}\end{array}$ & & \\
\hline 1.1 & 151 & 81 & 0.54 & 16.6 & 0.000054 & 0.09 & 7.817 & 0.128 & 0.0643 & 0.0014 & 0.1285 & 0.0021 & $\begin{array}{lll}1.204 & 0.033\end{array}$ & 0.0680 & 0.0015 & 0.605 & 779 & 13 & 868 & 45 & 10 \\
\hline 2.1 & 94 & 65 & 0.69 & 3.5 & 0.003061 & 2.89 & 23.141 & 0.656 & 0.0747 & 0.0030 & 0.0420 & 0.0012 & & & & & 265.0 & 7.5 & & & \\
\hline 3.1 & 181 & 142 & 0.79 & 15.8 & 0.000090 & 0.09 & 9.839 & 0.157 & 0.0613 & 0.0012 & 0.1015 & 0.0017 & & & & & 623.5 & 9.7 & & & \\
\hline 4.1 & 271 & 110 & 0.41 & 18.3 & 0.000008 & 0.27 & 12.717 & 0.185 & 0.0591 & 0.0011 & 0.0784 & 0.0012 & & & & & 486.7 & 7.0 & & & \\
\hline 5.1 & 1373 & 229 & 0.17 & 49.4 & - & $<0.01$ & 23.893 & 0.287 & 0.0511 & 0.0006 & 0.0419 & 0.0005 & & & & & 264.5 & 3.1 & & & \\
\hline 6.1 & 181 & 91 & 0.50 & 5.9 & - & 0.12 & 26.192 & 0.499 & 0.0519 & 0.0027 & 0.0381 & 0.0007 & & & & & 241.3 & 4.6 & & & \\
\hline 7.1 & 112 & 25 & 0.22 & 9.0 & 0.000168 & 0.57 & 10.769 & 0.233 & 0.0637 & 0.0017 & 0.0923 & 0.0020 & & & & & 569.3 & 12.1 & & & \\
\hline 8.1 & 185 & 154 & 0.83 & 6.2 & - & 0.25 & 25.483 & 0.459 & 0.0531 & 0.0017 & 0.0391 & 0.0007 & & & & & 247.5 & 4.4 & & & \\
\hline 9.1 & 192 & 247 & 1.29 & 6.8 & 0.000052 & 0.10 & 24.269 & 0.437 & 0.0522 & 0.0020 & 0.0412 & 0.0008 & & & & & 260.0 & 4.7 & & & \\
\hline 10.1 & 110 & 51 & 0.46 & 5.5 & 0.000295 & 0.33 & 17.125 & 0.346 & 0.0565 & 0.0019 & 0.0582 & 0.0012 & & & & & 364.7 & 7.3 & & & \\
\hline 11.1 & 51 & 11 & 0.22 & 3.0 & 0.001524 & 0.00 & 14.783 & 0.394 & 0.0552 & 0.0026 & 0.0676 & 0.0018 & & & & & 422.0 & 11.1 & & & \\
\hline 12.1 & 843 & 1144 & 1.36 & 29.9 & 0.000157 & 0.05 & 24.246 & 0.295 & 0.0519 & 0.0011 & 0.0412 & 0.0005 & & & & & 260.4 & 3.1 & & & \\
\hline 13.1 & 109 & 144 & 1.32 & 4.0 & 0.001446 & 0.24 & 23.344 & 0.501 & 0.0535 & 0.0021 & 0.0427 & 0.0009 & & & & & 269.8 & 5.8 & & & \\
\hline 14.1 & 175 & 23 & 0.13 & 13.0 & 0.000089 & $<0.01$ & 11.537 & 0.187 & 0.0575 & 0.0012 & 0.0867 & 0.0014 & & & & & 536.2 & 8.5 & & & \\
\hline 15.1 & 250 & 333 & 1.33 & 8.7 & - & 0.14 & 24.794 & 0.404 & 0.0524 & 0.0014 & 0.0403 & 0.0007 & & & & & 254.5 & 4.1 & & & \\
\hline 16.1 & 388 & 48 & 0.12 & 29.1 & - & 0.05 & 11.482 & 0.153 & 0.0586 & 0.0011 & 0.0871 & 0.0012 & & & & & 538.1 & 7.0 & & & \\
\hline 17.1 & 532 & 211 & 0.40 & 28.8 & - & $<0.01$ & 15.856 & 0.201 & 0.0530 & 0.0013 & 0.0632 & 0.0008 & & & & & 395.0 & 5.0 & & & \\
\hline 18.1 & 396 & 286 & 0.72 & 29.5 & 0.000129 & 0.32 & 11.514 & 0.152 & 0.0608 & 0.0009 & 0.0866 & 0.0012 & & & & & 535.2 & 6.9 & & & \\
\hline 19.1 & 96 & 74 & 0.77 & 3.4 & 0.001063 & 0.57 & 24.105 & 0.541 & 0.0560 & 0.0023 & 0.0412 & 0.0009 & & & & & 260.6 & 5.8 & & & \\
\hline 20.1 & 135 & 80 & 0.59 & 5.1 & - & $<0.01$ & 22.890 & 0.515 & 0.0496 & 0.0018 & 0.0438 & 0.0010 & & & & & 276.4 & 6.2 & & & \\
\hline 21.1 & 296 & 129 & & 15.3 & - & $<0.01$ & 16.593 & 0.237 & 0.0536 & 0.0011 & 0.0603 & 0.0009 & & & & & 377.5 & 5.3 & & & \\
\hline 22.1 & 497 & 713 & 1.43 & 17.5 & - & $<0.01$ & 24.390 & 0.324 & 0.0509 & 0.0010 & 0.0410 & 0.0006 & & & & & 259.2 & 3.4 & & & \\
\hline 23.1 & 197 & 164 & 0.83 & 6.9 & 0.000282 & 0.46 & 24.517 & 0.420 & 0.0550 & 0.0016 & 0.0406 & 0.0007 & & & & & 256.6 & 4.4 & & & \\
\hline 24.1 & 176 & 187 & 1.06 & 6.2 & 0.001437 & 0.08 & 24.459 & 0.438 & 0.0521 & 0.0016 & 0.0409 & 0.0007 & & & & & 258.1 & 4.6 & & & \\
\hline 25.1 & 258 & 246 & 0.95 & 8.6 & - & 0.20 & 25.889 & 0.415 & 0.0527 & 0.0014 & 0.0385 & 0.0006 & & & & & 243.8 & 3.9 & & & \\
\hline 26.1 & 139 & 155 & 1.12 & 4.7 & 0.001072 & 0.35 & 25.520 & 0.503 & 0.0539 & 0.0020 & 0.0390 & 0.0008 & & & & & 246.9 & 4.8 & & & \\
\hline 27.1 & 168 & 119 & 0.71 & 5.5 & - & 0.10 & 26.433 & 0.493 & 0.0518 & 0.0020 & 0.0378 & 0.0007 & & & & & 239.1 & 4.4 & & & \\
\hline 28.1 & 287 & 206 & 0.72 & 9.7 & - & 0.42 & 25.287 & 0.392 & 0.0546 & 0.0014 & 0.0394 & 0.0006 & & & & & 249.0 & 3.8 & & & \\
\hline 29.1 & 133 & 127 & 0.95 & 12.4 & 0.000038 & $<0.01$ & 9.222 & 0.157 & 0.0603 & 0.0014 & 0.1086 & 0.0019 & & & & & 664.8 & 11.0 & & & \\
\hline 30.1 & 129 & 106 & 0.82 & 10.4 & 0.000342 & 0.18 & 10.622 & 0.209 & 0.0608 & 0.0014 & 0.0940 & 0.0019 & & & & & 579.0 & 11.1 & & & \\
\hline 31.1 & 89 & 94 & 1.06 & 3.1 & - & 0.17 & 24.235 & 0.556 & 0.0528 & 0.0024 & 0.0412 & 0.0010 & & & & & 260.2 & 5.9 & & & \\
\hline 32.1 & 259 & 44 & 0.17 & 18.7 & 0.000125 & 0.21 & 11.907 & 0.169 & 0.0594 & 0.0010 & 0.0838 & 0.0012 & & & & & 518.8 & 7.2 & & & \\
\hline 33.1 & 201 & 97 & 0.49 & 14.0 & 0.000109 & $<0.01$ & 12.354 & 0.188 & 0.0572 & 0.0012 & 0.0810 & 0.0013 & & & & & 501.8 & 7.5 & & & \\
\hline 34.1 & 461 & 293 & 0.64 & 35.9 & 0.000041 & $<0.01$ & 11.047 & 0.138 & 0.0576 & 0.0007 & 0.0907 & 0.0012 & & & & & 559.4 & 6.8 & & & \\
\hline 35.1 & 535 & 443 & 0.83 & 18.5 & 0.000022 & 0.22 & 24.865 & 0.323 & 0.0530 & 0.0013 & 0.0401 & 0.0005 & & & & & 253.6 & 3.3 & & & \\
\hline 36.1 & 123 & 91 & 0.74 & 8.0 & - & 0.06 & 13.133 & 0.237 & 0.0570 & 0.0015 & 0.0761 & 0.0014 & & & & & 472.8 & 8.4 & & & \\
\hline 37.1 & 149 & 173 & 1.16 & 5.3 & 0.000630 & 0.58 & 24.320 & 0.451 & 0.0561 & 0.0018 & 0.0409 & 0.0008 & & & & & 258.3 & 4.8 & & & \\
\hline 38.1 & 130 & 128 & 0.98 & 4.9 & - & 0.31 & 23.040 & 0.443 & 0.0542 & 0.0019 & 0.0433 & 0.0008 & & & & & 273.1 & 5.2 & & & \\
\hline 39.1 & 457 & 436 & 0.96 & 16.2 & - & $<0.01$ & 24.265 & 0.361 & 0.0513 & 0.0010 & 0.0412 & 0.0006 & & & & & 260.4 & 3.8 & & & \\
\hline 40.1 & 410 & 201 & 0.49 & 31.0 & 0.000206 & 0.09 & 11.354 & 0.144 & 0.0591 & 0.0010 & 0.0880 & 0.0011 & & & & & 543.6 & 6.8 & & & \\
\hline 41.1 & 522 & 410 & 0.79 & 19.1 & 0.000260 & 0.55 & 23.473 & 0.299 & 0.0560 & 0.0009 & 0.0424 & 0.0005 & & & & & 267.5 & 3.4 & & & \\
\hline 42.1 & 806 & 471 & 0.58 & 39.8 & 0.000009 & 0.16 & 17.373 & 0.203 & 0.0550 & 0.0006 & 0.0575 & 0.0007 & & & & & 360.2 & 4.1 & & & \\
\hline 43.1 & 173 & 106 & 0.61 & 15.3 & 0.000052 & 0.29 & 9.714 & 0.150 & 0.0631 & 0.0012 & 0.1026 & 0.0016 & & & & & 629.9 & 9.5 & & & \\
\hline 44.1 & 556 & 898 & 1.61 & 35.4 & 0.000099 & 0.42 & 13.511 & 0.164 & 0.0596 & 0.0007 & 0.0737 & 0.0009 & & & & & 458.4 & 5.5 & & & \\
\hline
\end{tabular}


Table 2 (continued)

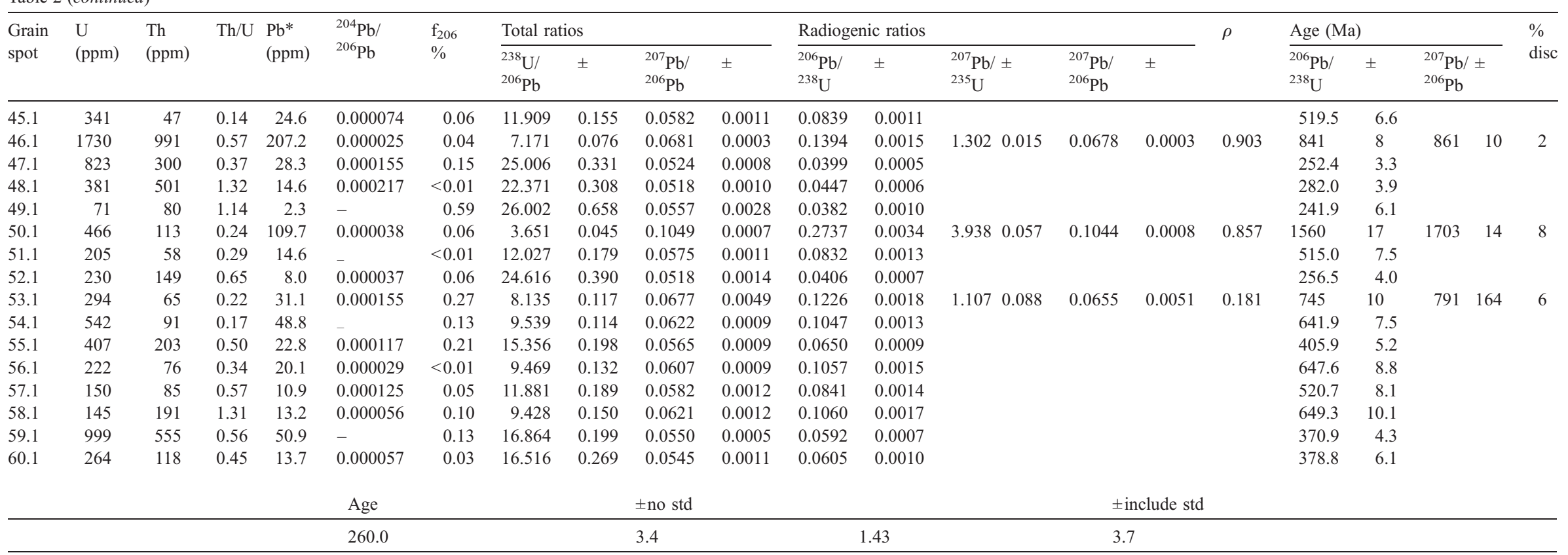

Notes :

1. Uncertainties given at the one $\sigma$ level.

2. Error in FC1 Reference zircon calibration was $0.59 \%$ for the analytical session. (not included in above errors but required when comparing ${ }^{206} \mathrm{~Pb} /{ }^{238} \mathrm{U}$ data from different mounts).

3. $\mathrm{f}_{206} \%$ denotes the percentage of ${ }^{206} \mathrm{~Pb}$ that is common $\mathrm{Pb}$.

4. For areas older than $\sim 800 \mathrm{Ma}$ correction for common $\mathrm{Pb}$ made using the measured ${ }^{204} \mathrm{~Pb} /{ }^{206} \mathrm{~Pb}$ ratio.

5. For areas younger than $\sim 800 \mathrm{Ma}$ correction for common $\mathrm{Pb}$ made using the measured ${ }^{238} \mathrm{U} /{ }^{206} \mathrm{~Pb}$ and ${ }^{207} \mathrm{~Pb} /{ }^{206} \mathrm{~Pb}$ ratios following Tera and Wasserburg (1972) as outlined in Williams (1998).

6 . For $\%$ disc, $0 \%$ denotes a concordant analysis.

$\mathrm{Pb}^{*}$ indicates radiogenic $\mathrm{Pb}$. 
Table 3

Summary of SHRIMP U-Pb zircon results for sample 96-36-4

\begin{tabular}{|c|c|c|c|c|c|c|c|c|c|c|c|c|c|c|c|c|c|c|c|c|c|c|}
\hline \multirow{2}{*}{$\begin{array}{l}\text { Grain } \\
\text { spot }\end{array}$} & \multirow{2}{*}{$\begin{array}{l}\mathrm{U} \\
(\mathrm{ppm})\end{array}$} & \multirow{2}{*}{$\begin{array}{l}\text { Th } \\
\text { (ppm) }\end{array}$} & \multirow[t]{2}{*}{$\mathrm{Th} / \mathrm{U}$} & \multirow{2}{*}{$\begin{array}{l}\mathrm{Pb}^{*} \\
\text { (ppm) }\end{array}$} & \multirow{2}{*}{$\begin{array}{l}{ }^{204} \mathrm{~Pb} / \\
{ }^{206} \mathrm{~Pb}\end{array}$} & \multirow{2}{*}{$\begin{array}{l}\mathrm{f}_{206} \\
\%\end{array}$} & \multicolumn{4}{|c|}{ Total ratios } & \multicolumn{6}{|c|}{ Radiogenic ratios } & \multirow[t]{2}{*}{$\rho$} & \multicolumn{4}{|c|}{ Age (Ma) } & \multirow{2}{*}{$\begin{array}{l}\% \\
\text { disc }\end{array}$} \\
\hline & & & & & & & $\begin{array}{l}{ }^{238} \mathrm{U} / \\
{ }^{206} \mathrm{~Pb}\end{array}$ & \pm & $\begin{array}{l}{ }^{207} \mathrm{~Pb} / \\
{ }^{206} \mathrm{~Pb}\end{array}$ & \pm & $\begin{array}{l}{ }^{206} \mathrm{~Pb} / \\
{ }^{238} \mathrm{U}\end{array}$ & \pm & $\begin{array}{l}{ }^{207} \mathrm{~Pb} / \\
{ }^{235} \mathrm{U}\end{array}$ & \pm & $\begin{array}{l}{ }^{207} \mathrm{~Pb} / \\
{ }^{206} \mathrm{~Pb}\end{array}$ & \pm & & $\begin{array}{l}{ }^{206} \mathrm{~Pb} / \\
{ }^{238} \mathrm{U}\end{array}$ & \pm & $\begin{array}{l}{ }^{207} \mathrm{~Pb} / \\
{ }^{206} \mathrm{~Pb}\end{array}$ & \pm & \\
\hline 1.1 & 579 & 30 & 0.05 & 72.0 & 0.000164 & 0.28 & 6.911 & 0.091 & 0.0737 & 0.0007 & 0.1443 & 0.0019 & 1.420 & 0.033 & 0.0714 & 0.0014 & 0.573 & 869 & 11 & 969 & 39 & 10 \\
\hline 2.1 & 91 & 80 & 0.87 & 23.0 & - & $<0.01$ & 3.417 & 0.059 & 0.1005 & 0.0014 & 0.2934 & 0.0050 & 4.153 & 0.092 & 0.1027 & 0.0014 & 0.774 & 1659 & 29 & 1673 & 26 & 1 \\
\hline 3.1 & 58 & 19 & 0.33 & 2.0 & - & 1.00 & 24.977 & 0.688 & 0.0592 & 0.0038 & 0.0396 & 0.0011 & & & & & & 250.6 & 6.9 & & & \\
\hline 4.1 & 66 & 40 & 0.61 & 9.9 & - & $<0.01$ & 5.686 & 0.114 & 0.0738 & 0.0018 & 0.1763 & 0.0036 & 1.848 & 0.079 & 0.0760 & 0.0029 & 0.471 & 1047 & 20 & 1096 & 76 & 4 \\
\hline 5.1 & 77 & 16 & 0.21 & 6.9 & - & 0.10 & 9.619 & 0.206 & 0.0617 & 0.0022 & 0.1039 & 0.0023 & & & & & & 637.0 & 13.4 & & & \\
\hline 6.1 & 412 & 331 & 0.80 & 32.9 & - & $<0.01$ & 10.750 & 0.153 & 0.0590 & 0.0009 & 0.0930 & 0.0014 & & & & & & 573.5 & 8.0 & & & \\
\hline 7.1 & 249 & 176 & 0.71 & 19.9 & 0.000466 & 0.28 & 10.745 & 0.157 & 0.0614 & 0.0011 & 0.0928 & 0.0014 & & & & & & 572.2 & 8.2 & & & \\
\hline 8.1 & 295 & 164 & 0.56 & 21.8 & 0.000200 & $<0.01$ & 11.604 & 0.178 & 0.0559 & 0.0010 & 0.0864 & 0.0014 & & & & & & 534.3 & 8.0 & & & \\
\hline 9.1 & 337 & 123 & 0.37 & 25.8 & 0.000150 & 0.06 & 11.232 & 0.155 & 0.0590 & 0.0010 & 0.0890 & 0.0013 & & & & & & 549.5 & 7.4 & & & \\
\hline 10.1 & 72 & 25 & 0.35 & 22.5 & - & $<0.01$ & 2.741 & 0.051 & 0.1809 & 0.0021 & 0.3650 & 0.0067 & 9.128 & 0.200 & 0.1814 & 0.0021 & 0.844 & 2006 & 32 & 2665 & 19 & 25 \\
\hline 11.1 & 101 & 58 & 0.58 & 6.7 & - & $<0.01$ & 12.990 & 0.261 & 0.0548 & 0.0020 & 0.0772 & 0.0016 & & & & & & 479.1 & 9.5 & & & \\
\hline 12.1 & 482 & 409 & 0.85 & 32.1 & - & 0.07 & 12.893 & 0.169 & 0.0573 & 0.0008 & 0.0775 & 0.0010 & & & & & & 481.2 & 6.2 & & & \\
\hline 13.1 & 104 & 51 & 0.49 & 8.4 & - & 0.44 & 10.687 & 0.196 & 0.0628 & 0.0018 & 0.0932 & 0.0018 & & & & & & 574.2 & 10.4 & & & \\
\hline 14.1 & 473 & 117 & 0.25 & 33.4 & - & $<0.01$ & 12.164 & 0.160 & 0.0564 & 0.0008 & 0.0823 & 0.0011 & & & & & & 509.9 & 6.6 & & & \\
\hline 15.1 & 180 & 77 & 0.43 & 28.4 & 0.000162 & 0.27 & 5.450 & 0.081 & 0.0762 & 0.0010 & 0.1841 & 0.0027 & 2.004 & 0.041 & 0.0790 & 0.0011 & 0.731 & 1089 & 16 & 1171 & 27 & 7 \\
\hline 16.1 & 94 & 57 & 0.60 & 6.9 & 0.000394 & 0.16 & 11.650 & 0.239 & 0.0593 & 0.0021 & 0.0857 & 0.0018 & & & & & & 530.1 & 10.7 & & & \\
\hline 17.1 & 194 & 87 & 0.45 & 17.6 & 0.000048 & $<0.01$ & 9.453 & 0.156 & 0.0599 & 0.0012 & 0.1060 & 0.0018 & & & & & & 649.3 & 10.5 & & & \\
\hline 18.1 & 177 & 138 & 0.78 & 15.6 & 0.000215 & 0.03 & 9.717 & 0.153 & 0.0610 & 0.0013 & 0.1029 & 0.0017 & & & & & & 631.3 & 9.7 & & & \\
\hline 19.1 & 339 & 122 & 0.36 & 17.0 & 0.000212 & 0.24 & 17.166 & 0.257 & 0.0558 & 0.0013 & 0.0581 & 0.0009 & & & & & & 364.2 & 5.4 & & & \\
\hline 20.1 & 206 & 123 & 0.60 & 14.5 & - & 0.19 & 12.211 & 0.185 & 0.0589 & 0.0014 & 0.0817 & 0.0013 & & & & & & 506.5 & 7.5 & & & \\
\hline 21.1 & 285 & 159 & 0.56 & 23.2 & - & 0.08 & 10.570 & 0.149 & 0.0601 & 0.0010 & 0.0945 & 0.0014 & & & & & & 582.3 & 8.1 & & & \\
\hline 22.1 & 137 & 69 & 0.51 & 11.2 & - & 0.31 & 10.500 & 0.173 & 0.0620 & 0.0015 & 0.0949 & 0.0016 & & & & & & 584.7 & 9.4 & & & \\
\hline 23.1 & 101 & 53 & 0.53 & 9.7 & 0.000100 & 7.07 & 8.901 & 0.159 & 0.1189 & 0.0149 & 0.1044 & 0.0030 & & & & & & 640.2 & 17.5 & & & \\
\hline 24.1 & 178 & 143 & 0.80 & 7.1 & 0.000081 & $<0.01$ & 21.536 & 0.387 & 0.0487 & 0.0017 & 0.0466 & 0.0009 & & & & & & 293.8 & 5.2 & & & \\
\hline 25.1 & 45 & 1 & 0.01 & 3.4 & - & 0.23 & 11.289 & 0.313 & 0.0603 & 0.0027 & 0.0884 & 0.0025 & & & & & & 545.9 & 14.9 & & & \\
\hline 26.1 & 147 & 53 & 0.36 & 6.9 & 0.000074 & $<0.01$ & 18.353 & 0.329 & 0.0523 & 0.0016 & 0.0546 & 0.0010 & & & & & & 342.4 & 6.1 & & & \\
\hline 27.1 & 765 & 277 & 0.36 & 35.3 & 0.000078 & 0.07 & 18.620 & 0.224 & 0.0538 & 0.0007 & 0.0537 & 0.0007 & & & & & & 337.0 & 4.0 & & & \\
\hline 28.1 & 114 & 94 & 0.83 & 8.9 & 0.000040 & 0.13 & 11.029 & 0.203 & 0.0598 & 0.0015 & 0.0906 & 0.0017 & & & & & & 558.8 & 10.1 & & & \\
\hline 29.1 & 287 & 81 & 0.28 & 21.8 & 0.000307 & 0.12 & 11.281 & 0.161 & 0.0594 & 0.0010 & 0.0885 & 0.0013 & & & & & & 546.9 & 7.6 & & & \\
\hline 30.1 & 255 & 140 & 0.55 & 11.6 & 0.000034 & 0.22 & 18.992 & 0.306 & 0.0548 & 0.0014 & 0.0525 & 0.0009 & & & & & & 330.1 & 5.3 & & & \\
\hline 31.1 & 209 & 155 & 0.74 & 16.8 & 0.000039 & 0.38 & 10.666 & 0.166 & 0.0624 & 0.0012 & 0.0934 & 0.0015 & & & & & & 575.6 & 8.8 & & & \\
\hline 32.1 & 111 & 76 & 0.68 & 7.7 & 0.000153 & 0.07 & 12.424 & 0.244 & 0.0578 & 0.0018 & 0.0804 & 0.0016 & & & & & & 498.7 & 9.6 & & & \\
\hline 33.1 & 343 & 163 & 0.48 & 26.6 & 0.001117 & 2.26 & 11.104 & 0.149 & 0.0768 & 0.0010 & 0.0880 & 0.0012 & & & & & & 543.8 & 7.3 & & & \\
\hline 34.1 & 72 & 61 & 0.85 & 2.8 & 0.000381 & 0.67 & 22.395 & 0.573 & 0.0573 & 0.0027 & 0.0444 & 0.0012 & & & & & & 279.8 & 7.1 & & & \\
\hline 35.1 & 90 & 46 & 0.52 & 6.9 & 0.000862 & 0.19 & 11.189 & 0.225 & 0.0601 & 0.0018 & 0.0892 & 0.0018 & & & & & & 550.8 & 10.9 & & & \\
\hline 36.1 & 96 & 72 & 0.75 & 6.6 & - & $<0.01$ & 12.518 & 0.256 & 0.0570 & 0.0018 & 0.0799 & 0.0017 & & & & & & 495.5 & 10.0 & & & \\
\hline 37.1 & 209 & 122 & 0.58 & 17.4 & 0.000013 & $<0.01$ & 10.314 & 0.180 & 0.0579 & 0.0011 & 0.0972 & 0.0017 & & & & & & 597.9 & 10.2 & & & \\
\hline 38.1 & 361 & 284 & 0.79 & 25.6 & 0.001273 & 2.12 & 12.121 & 0.164 & 0.0744 & 0.0011 & 0.0808 & 0.0011 & & & & & & 500.6 & 6.8 & & & \\
\hline 39.1 & 405 & 207 & 0.51 & 48.0 & 0.000033 & 0.06 & 7.257 & 0.093 & 0.0693 & 0.0008 & 0.1377 & 0.0018 & 1.307 & 0.023 & 0.0688 & 0.0008 & 0.723 & 832 & 10 & 894 & 25 & 7 \\
\hline 40.1 & 355 & 106 & 0.30 & 33.7 & - & 0.06 & 9.057 & 0.119 & 0.0625 & 0.0008 & 0.1103 & 0.0015 & & & & & & 674.7 & 8.6 & & & \\
\hline 42.1 & 196 & 75 & 0.39 & 12.7 & - & 0.29 & 13.225 & 0.216 & 0.0588 & 0.0014 & 0.0754 & 0.0013 & & & & & & 468.6 & 7.5 & & & \\
\hline 43.1 & 61 & 22 & 0.36 & 24.0 & 0.000061 & 0.09 & 2.178 & 0.045 & 0.1747 & 0.0019 & 0.4586 & 0.0095 & 11.002 & 0.278 & 0.1740 & 0.0025 & 0.821 & 2434 & 42 & 2596 & 24 & 6 \\
\hline 44.1 & 323 & 161 & 0.50 & 31.9 & 0.000128 & 0.87 & 8.699 & 0.118 & 0.0698 & 0.0009 & 0.1139 & 0.0016 & & & & & & 695.6 & 9.3 & & & \\
\hline 45.1 & 323 & 130 & 0.40 & 34.2 & 0.000189 & 0.20 & 8.097 & 0.111 & 0.0659 & 0.0009 & 0.1233 & 0.0017 & & & & & & 749.3 & 10.0 & & & \\
\hline
\end{tabular}


Table 3 (continued)

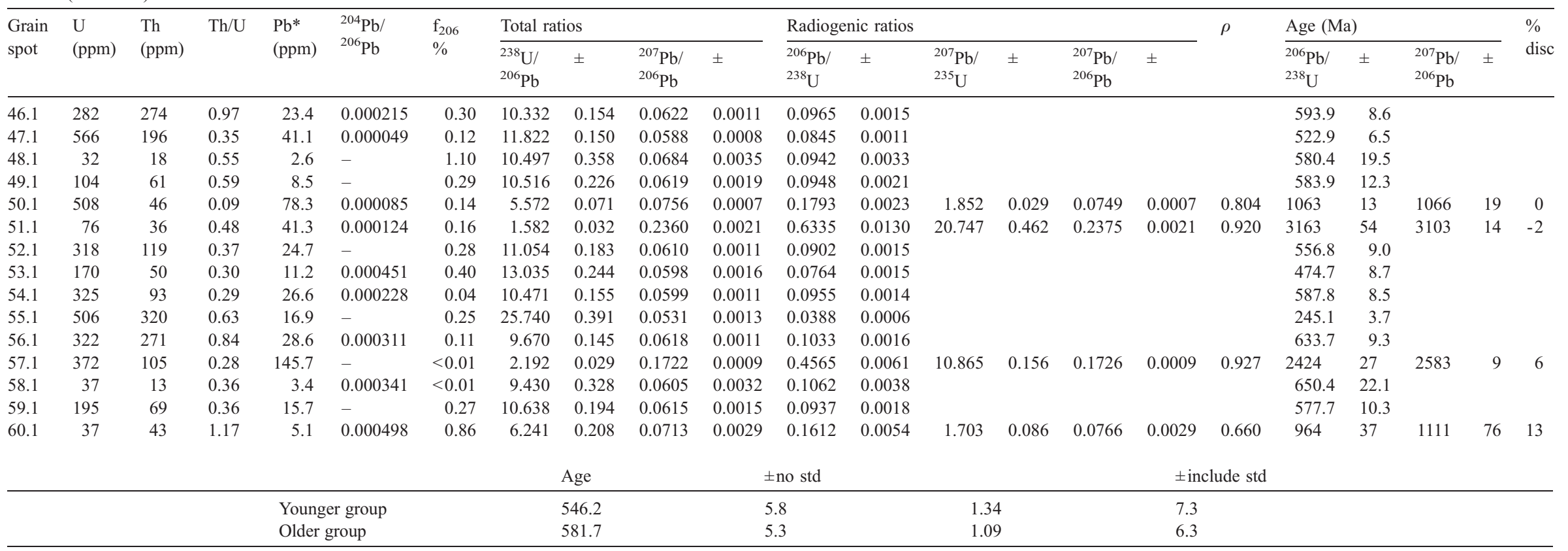

Notes :

1. Uncertainties given at the one $\sigma$ level.

2. Error in FC1 Reference zircon calibration was $0.59 \%$ for the analytical session. (not included in above errors but required when comparing ${ }^{206} \mathrm{~Pb}{ }^{238} \mathrm{U}$ data from different mounts).

3. $\mathrm{f}_{206} \%$ denotes the percentage of ${ }^{206} \mathrm{~Pb}$ that is common $\mathrm{Pb}$.

4. For areas older than $\sim 800 \mathrm{Ma}$ correction for common $\mathrm{Pb}$ made using the measured ${ }^{204} \mathrm{~Pb} /{ }^{206} \mathrm{~Pb}$ ratio.

5. For areas younger than $\sim 800 \mathrm{Ma}$ correction for common $\mathrm{Pb}$ made using the measured ${ }^{238} \mathrm{U} /{ }^{206} \mathrm{~Pb}$ and ${ }^{207} \mathrm{~Pb} / 206 \mathrm{~Pb}$ ratios following Tera and Wasserburg (1972) as outlined in Williams (1998).

6. For $\%$ disc, $0 \%$ denotes a concordant analysis.

$\mathrm{Pb}^{*}$ indicates radiogenic $\mathrm{Pb}$. 
but shows only laumontite grade metamorphism. Although the Buckley-Fremouw contact has long been considered the PermoTriassic boundary (Collinson et al., 1994; Retallack et al., 1998; Krull and Retallack, 2000), in the Shackleton Glacier region it is now known to lie within the lowest part of the Fremouw Formation in a 7-10 $\mathrm{m}$ interval $35-45 \mathrm{~m}$ above the BuckleyFremouw contact, above the last occurrence of Glossopteris and below the first Triassic vertebrates (Collinson et al., 2006).

\section{Methods}

Detrital zircon grains were separated from three middle Victoria Group sandstone samples using standard crushing, washing, heavy liquid (Sp. Gr. 2.96 and 3.3), and paramagnetic procedures. The zircon-rich heavy mineral concentrates were poured onto double-sided tape, mounted in epoxy together with chips of the reference zircons (FC1 and SL13), sectioned approximately in half, and polished. Reflected and transmitted light photomicrographs were prepared for all zircons, as were cathodoluminescence (CL) and Scanning Electron Microscope (SEM) images. The CL images were used to decipher the internal structures of the sectioned grains and to ensure that the $\sim 30 \mu \mathrm{m}$ SHRIMP spot was wholly within a single age component (usually the youngest).

The U-Th-Pb analyses were made using SHRIMP I at the Research School of Earth Sciences, Australian National University, Canberra, Australia. For each sample, zircons on the mount were analyzed sequentially and randomly until a total of at least 60 grains for each sample was reached (Vermeesch, 2004). Each analysis consists of 4 scans through the mass range, with the FC1 reference zircon analyzed for every five unknown zircon analyses; SHRIMP analytical methods follow Williams (1998) (and the references therein). The data have been reduced using the SQUID Excel Macro of Ludwig (2001). U/Pb ratios have been normalized relative to a value of 0.01859 for the FC1 reference zircon, equivalent to an age of $1099 \mathrm{Ma}$ (see Paces and Miller, 1993). Uncertainties given for individual analyses (ratios and ages) are at the one sigma level (Tables 1, 2 and 3). Tera and Wasserburg (1972) concordia plots, probability density plots with stacked histograms and weighted mean ${ }^{206} \mathrm{~Pb} /{ }^{238} \mathrm{U}$ age calculations were carried out using ISOPLOT/ EX (Ludwig, 2003). The "Mixture Modeling" algorithm of Sambridge and Compston (1994), via ISOPLOT/EX, was used to un-mix statistical age populations or groupings; from these groups weighted mean ${ }^{206} \mathrm{~Pb} /{ }^{238} \mathrm{U}$ ages were calculated and the uncertainties are reported as $95 \%$ confidence limits.

\section{Results}

Sample 35-2 (ca. $70 \mathrm{~m}$ below the Buckley-Fremouw contact). The zircons from this sample are notably euhedral, or fragments of euhedral, doubly terminated grains with simple oscillatoryzoned internal structure when viewed by CL (Fig. 5a). Only two grains show significant external rounding (grains 9 and 20) and these provide the only two older ages at ca.1185 Ma and ca. 685 Ma respectively (Table 1). Note that from the CL images these also are considered to be zoned igneous zircon. The relative probability plot shows almost exclusively late Permian zircons (Fig. 6), with the principal peak at 267 \pm 3 Ma and a subsidiary peak at $249 \pm 3 \mathrm{Ma}$. Four grains with ${ }^{206} \mathrm{~Pb} /{ }^{238} \mathrm{U}$ ages less than $240 \mathrm{Ma}$ do not form a coherent peak and are interpreted to be analyses of areas within grains that have lost radiogenic $\mathrm{Pb}$. The subordinate peak at ca. $250 \mathrm{Ma}$ is within uncertainty of the Permian-Triassic boundary $(252.6 \pm 0.2 \mathrm{Ma}$; Mundil et al., 2004), but those grains also may have suffered radiogenic $\mathrm{Pb}$
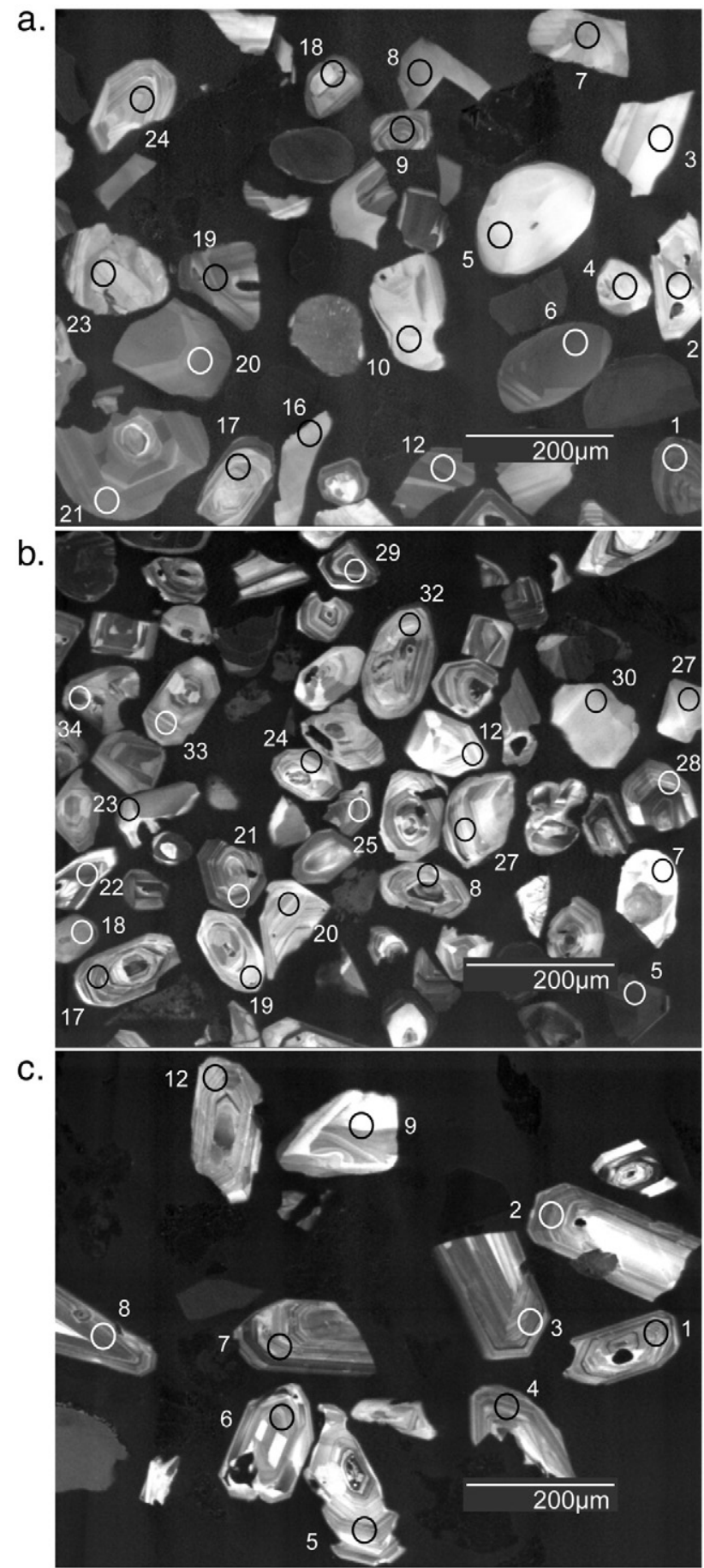

Fig. 5. Representative cathodoluminescence (CL) images for zircons analyzed from samples: a. 96-35-2; b. 96-36-1; c. 96-36-4. Note: areas analyzed are labeled with spot analysis numbers as in Tables 1,2 and 3. 


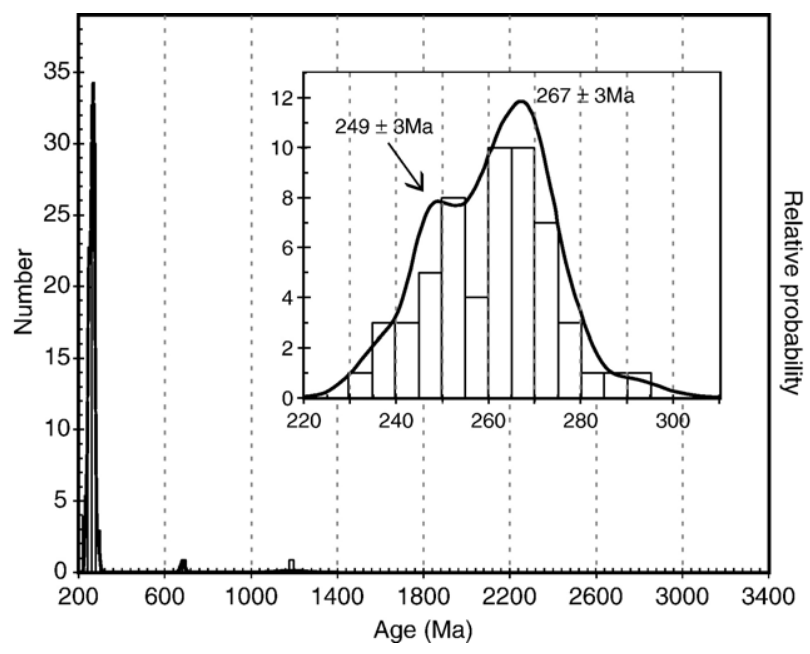

Fig. 6. U-Pb zircon age probability plot for sample 35-2, Layman Peak.

loss. The dominant $267 \pm 3$ Ma peak documents an abundance of grains from a magmatic source with essentially no dilution, during transport, from any sources with zircons of widely different ages. It should be noted that this sample and sample 36-1 are from the Buckley Formation which is characterized by the Permian plant fossil Glossopteris; Triassic ages cannot be given significance without further study.

Sample 36-1 (3 m below the Buckley-Fremouw contact). The zircons from this sample are equant to elongate grains that in general are subrounded to euhedral, or fragments of such grains. The CL images show a high proportion of zoned igneous zircon (Fig. 5b). Eleven of the 60 grains analyzed are considered to have metamorphic affinities (that is, homogeneous unzoned CL features) and these are not restricted solely to the older age components. The predominant population of grains is late Permian in age with a probability peak at $260 \pm 3.4 \mathrm{Ma}$ and a subordinate younger grouping at about $245 \mathrm{Ma}$ (Fig. 7; Table 2). It is probable that radiogenic $\mathrm{Pb}$ loss has affected the younger grains comprising this weak shoulder on the dominant peak. With respect to the remainder of the detrital age spectrum, there

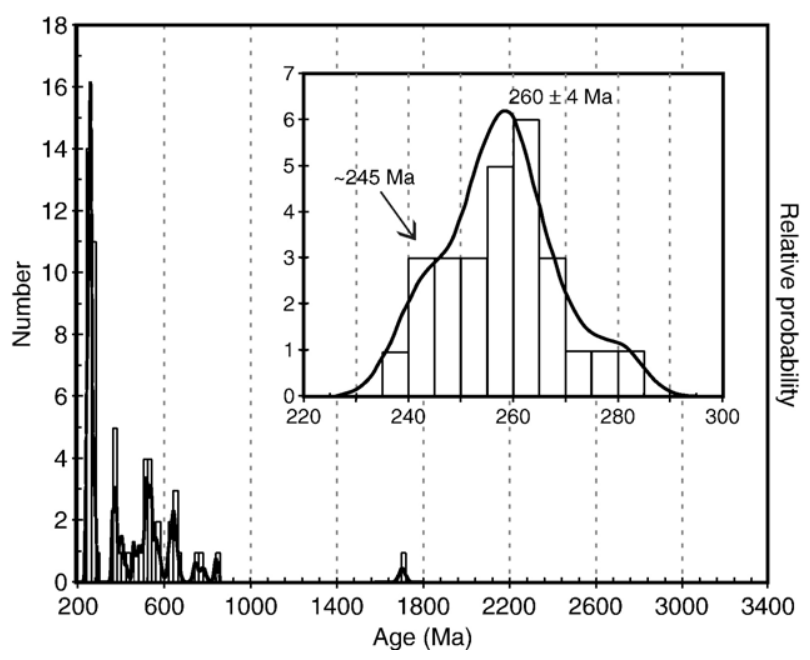

Fig. 7. U-Pb zircon age probability plot for sample 36-1, Collinson Ridge.

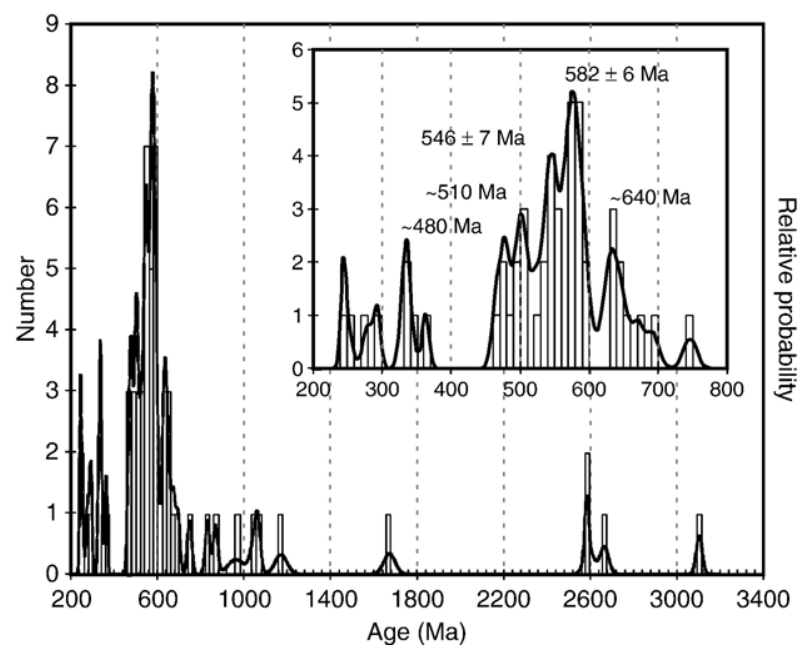

Fig. 8. U-Pb zircon age probability plot for sample 36-4, Collinson Ridge.

is an addition of late Devonian age grains with a peak at $370 \mathrm{Ma}$, and of early Cambrian Ross age or late Pan African age (540-510 Ma) and Neoproterozoic early Pan-African age $(\approx 640 \mathrm{Ma})$ grains.

Sample 36-4 (45 $\mathrm{m}$ above the Buckley-Fremouw contact). The zircons from this sample similarly are equant to elongate grains but in general are subrounded in shape, or fragments of such grains. Euhedral grain shapes are not common in this sample. Twenty four of the 60 grains analyzed have homogeneous internal structure, and are considered to be of a metamorphic provenance as determined from the CL images; the remainder are zoned igneous zircon (Fig. 5c). The metamorphic grains are spread throughout the total population, though they are more common among the Cambrian and older zircons. The age population is dominated by early Ordovician to Cambrian and by Neoproterozoic zircon grains, with four separate peaks (Fig. 8; Table 3). The 460-510 Ma group may reflect erosion of post-tectonic Ross granitoids. The 530-560 Ma group is consistent with erosion of pre-tectonic Ross granitoids or Pan African age rocks. The 570-600 Ma group forms a late Neoproterozoic source, which together with the $\approx 640 \mathrm{Ma}$ group comprise a Pan-African/East African Orogen age assemblage. There is also a small component of Carboniferous and Archean grains, and a scattering of poorly defined younger grains.

\section{Discussion}

The Permo-Triassic Gondwanide fold belt (Fig. 9), exemplified by the Cape Fold Belt in South Africa (e.g. Hälbich, 1992), is recorded in strong deformation of Permian and older strata in the displaced Ellsworth-Whitmore Mountains block (Curtis and Storey, 1996) and in gently deformed Permian strata at the distal margin of the belt in the Pensacola Mountains (Ford, 1972). Continuation of the foreland fold and thrust belt into the Ross Sea sector has been inferred and has been regarded as the cause of the major paleocurrent reversal in the PermoTriassic Victoria Group strata (Barrett et al., 1986; Collinson et al., 1994). 




Fig. 9. Gondwana reconstruction for the Antarctic sector, showing the inferred locations of orogenic belts, generalized distribution of dated plutonic rocks and orthogneisses, inferred extent of the Beacon Supergroup sedimentary basins, and the inferred Gondwana orogen structural front. All reconstructions have problems reconciling the geology, continental blocks, and space issues; this is exemplified by Tasmania which, in terms of its geology, should lie along the boundary between the Early and mid Paleozoic orogenic belts. CHP: Challenger Plateau, Lord Howe Rise, and New Zealand Paleozoic and older basement rocks; CP: Campbell Plateau, Chatham Rise, and New Zealand Paleozoic margin strata. Reconstruction modified from Fig. 1 of Elliot and Fleming (2004).

Paleocurrent indicators for the Victoria Group strata in the Shackleton Glacier region show detrital grain derivation from the West Antarctica flank of the foreland basin (Collinson et al.,
1994). The flood plain sequences reflect progradation, from mid Permian time onward, of alluvial settings from the arc flank into the basin, and a concomitant cratonward shift of the main axial drainage (Isbell et al., 1997).

The Permian zircons are igneous in origin and directly record a late Permian magmatic arc. The Devonian zircons, also igneous in origin, suggest derivation from Ford Granodiorite plutons in MBL and/or from an uncertain extension of that belt eastward toward the Antarctic Peninsula. Shifting source regions, reflecting tectonism and evolving paleodrainage systems, and/or deeper erosion down through the rising magmatic arc or its foreland probably account for the influx of Devonian zircons and the minor occurrence of Carboniferous grains.

More problematic is the presence of a major influx of Neoproterozoic and Cambrian zircon grains in the lowest Triassic sandstone (sample 36-4). In Permian and Early to Middle Triassic time, the foreland basin was separated from the intracratonic basins in Victoria Land by the Ross High located in the Darwin Glacier-Byrd Glacier region (Collinson et al., 1994). Thicknesses $(>1,000 \mathrm{~m})$ of Permian strata in the foreland basin suggest original continuation of the basin out onto the craton, although now eroded or hidden beneath the polar ice sheet, and also continuation of the basin toward the active margin in what is now West Antarctica. Permian foreland basin strata, and also the intracratonic basin strata of SVL, cover Devonian beds and the pre-Devonian basement; it is only north of the Dry Valley region of SVL that basement was exposed in late Permian through late Triassic time. Therefore, given detrital grain derivation from the magmatic arc flank, the pre-Devonian basement of the TAM could not have formed the immediate source for these late Proterozoic and Cambrian zircons. There is a single orthogneiss outcrop of possible Cambrian age in eastern MBL (Pankhurst et al., 1998), which suggests other gneiss and granitoid outcrops of that age might be present in the Ross Province, but no Proterozoic outcrops are known. However, detrital zircons from the Ordovician Swanson Group in MBL have an age population peak at 500-600 Ma and a subsidiary older peak at 800 $1000 \mathrm{Ma}$ (Pankhurst et al., 1998). Although the lowest Triassic sandstone sample lacks any significant number of grains in the $800-1000$ Ma age range, if the Swanson Formation originally had extended farther east, it could have been a contributory source for the Cambrian and latest Neoproterozoic grains.

Ross age granites occur along the inner flank of the TAM and may well be present farther inboard as well as in West Antarctica, the direction of younging of that plutonic belt (Goodge et al., 2004a). Devonian sandstones formed a shallow epicontinental sequence deposited along the length of the TAM

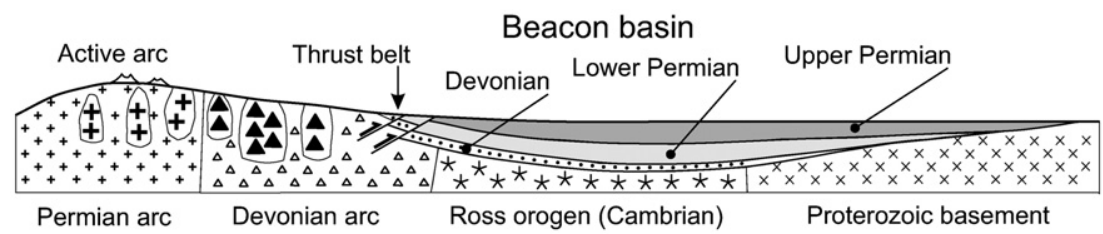

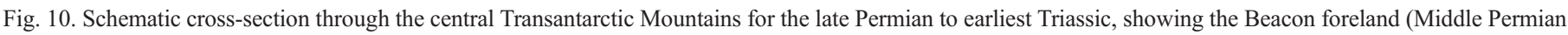

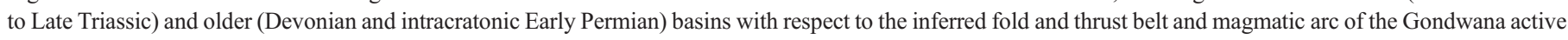
margin. 
but extending into West Antarctica (Bradshaw and Webers, 1988). The source terrain for the Devonian quartzose sandstones was the stable craton, and possibly Neoproterozoic and Cambro-Ordovician rocks involved in the Ross orogen. A Devonian Junction Sandstone sample in the Darwin Glacier region has yielded detrital zircons with the principal population peak dominated by 450-600 Ma grains, and minor peaks at 1.0-1.2 Ga, 1.4-1.7 Ga and 2.7 Ga (Wysoczanski et al., 1997, 2003). Further, assuming open connections in the Devonian epicontinental sea outboard of the TAM, zircons might also have been derived from more distal sources; this applies in particular to the Pan-African age zircons for which the nearest known source would have been near the head of the Weddell Sea (Fitzsimons, 2000, 2003) in the Shackleton Range where the East African Orogen appears to terminate. Lower Buckley and older Permian beds, deposited on the Devonian strata, also had non-volcanic sources (Collinson et al., 1994). Therefore the principal source for the late Proterozoic and Cambrian zircons is interpreted to have been Devonian and lower Permian strata and/or Swanson Group rocks, all exposed in the inferred extension of the Permo-Triassic Gondwanide fold and thrust belt (Fig. 10). The possibility of other, but no longer exposed, Lower Paleozoic and older sources in West Antarctica cannot be excluded. The younger populations indicate continuing erosion of mid Paleozoic plutonic rocks.

The appearance of these Cambrian and Neoproterozoic zircon populations in uppermost Permian and lowermost Triassic beds may mark the first record of erosion of the inferred thrust belt in this part of the Gondwanide orogen, although the onset of thrusting may well have been earlier in the Permian. In the Pensacola Mountains, Gondwana deformation can be dated only as post-Gale Mudstone tillites (Ford, 1972), and in the Ellsworth Mountains as post-Polarstar Formation or post-Middle Permian/earliest Late Permian (Collinson et al., 1994).

These zircon data suggest the need for revision of earlier interpretations (Barrett et al., 1986; Collinson et al., 1994) of Victoria Group quartzose sandstones as reflecting input primarily from a cratonic provenance into the foreland basin. Lower Buckley and older strata probably have a direct cratonic provenance but thereafter input from the active margin may have overwhelmed the sand provenance of the axial river system that flowed parallel to the range but received sediment from both the margin and craton. Further, sources of varying ages were being eroded in the active margin terrain, not just the contemporaneous Permo-Triassic magmatic arc.

\section{Conclusions}

Detrital zircons in the upper Buckley Formation and lower Fremouw Formation indicate several different source terrains within the Antarctic sector of the Permo-Triassic active margin of the Gondwana plate. The active magmatic arc was the principal provenance for the zircons in the Buckley volcaniclastic sandstones. The older mid Paleozoic magmatic arc granitoids contributed Devonian and Carboniferous detrital zircons as a result of unroofing of that arc and/or changes in paleo-drainage systems. It is inferred that active tectonism in the Gondwanide orogen exposed Devonian and Lower Permian strata and Ordovician rocks, which led to recycling of Neoproterozoic and Cambrian zircons into the Victoria Group foreland basin in latest Permian to early Triassic time. This study suggests an examination of the complete Beacon Supergroup sequence in CTM will shed light on sediment provenance and tectonic evolution of the Permo-Triassic foreland basin.

\section{Acknowledgments}

Field research was supported by NSF Grant OPP 95-27816 (DHE) and costs for laboratory analysis were supported by NSF Grant OPP 00-87919 (DHE). Jeff Ziga performed the initial zircon separations. Discussion of the Victoria Group with John Isbell and James Collinson is greatly appreciated. We thank John Isbell and Michael Palin for constructive reviews, as well as the Editor, Alan Vaughan. Byrd Polar Research Center Contribution No. 1353.

\section{References}

Adams, C.J., Seward, D., Weaver, S.D., 1995. Geochronology of Cretaceous granites and metasedimentary basement on Edward VII Peninsula, Marie Byrd Land, West Antarctica. Antarctic Science 7, 265-277.

Adams, C.J., Barley, M.E., Maas, R., Doyle, M.G., 2002. Provenance of Permian-Triassic volcaniclastic sedimentary terranes in New Zealand: evidence from their radiogenic isotope characteristics and detrital mineral age patterns. New Zealand Journal of Geology and Geophysics 45, 221-242.

Anderson, J.M., 1979. The geology of the Taylor Group, Beacon Supergroup, Byrd Glacier area, Antarctica. New Zealand Antarctic Record 2, 5-11.

Barrett, P.J., Elliot, D.H., Lindsay, J.F., 1986. The Beacon Supergroup (Devonian-Triassic) and Ferrar Group (Jurassic) in the Beardmore Glacier area, Antarctica. In: Turner, M.D., Splettstoesser, J.F. (Eds.), Geology of the central Transantarctic Mountains. American Geophysical Union, Antarctic Research Series, vol. 36, pp. 339-428.

Borg, S.G., DePaolo, D.J., Smith, B.M., 1990. Isotopic structure and tectonics of the central Transantarctic Mountains. Journal of Geophysical Research 95, $6647-6667$.

Bradshaw, M.A., 1991. The Devonian Pacific margin of Antarctica. In: Thomson, M.R.A., Crame, J.A., Thomson, J.W. (Eds.), Geological evolution of Antarctica. Cambridge University Press, Cambridge, pp. 193-197.

Bradshaw, M.A., Webers, G.F., 1988. The Devonian rocks of Antarctica. In: McMillan, N.J., Emery, A.F., Glass, D.J. (Eds.), Devonian of the World. Canadian Society of Petroleum Geology, Calgary, pp. 783-795.

Coates, D.A., 1985. Late Paleozoic glacial patterns in the central Transantarctic Mountains, Antarctica. In: Turner, M.D., Splettstoesser, J.F. (Eds.), Geology of the central Transantarctic Mountains. American Geophysical Union, Antarctic Research Series, vol. 36, pp. 325-338.

Collinson, J.W., Elliot, D.H., 1984. Triassic stratigraphy of the Shackleton Glacier area. In: Turner, M.D., Splettstoesser, J.F. (Eds.), Geology of the central Transantarctic Mountains. Antarctic Research Series, vol. 36 American Geophysical Union, pp. 103-117.

Collinson, J.W., Elliot, D.H., Isbell, J.L., Miller, J.M.G., 1994. PermianTriassic Transantarctic Basin. In: Veevers, J.J., Powell, C., Mc, A. (Eds.), Permian-Triassic Pangaean Basins and Foldbelts along the Panthalassan margin of Gondwanaland. Geological Society of America, Memoir 184. Boulder, Colorado, pp. 173-222.

Collinson, J.C., Hammer, W.R., Askin, R.A., Elliot, D.H., 2006. PermianTriassic boundary in the central Transantarctic Mountains, Antarctica. Geological Society of America Bulletin 118, 747-763.

Curtis, M.L., Storey, B.C., 1996. A review of geological constraints on the prebreak-up position of the Ellsworth Mountains within Gondwana: implications for Weddell Sea evolution. In: Storey, B.C., King, E.C., Livermore, R.A. 
(Eds.), Magmatism and the Causes of Continental Break-up. Special Publication, vol. 68. Geological Society of London, pp. 11-30.

Dalziel, I.W.D., Elliot, D.H., 1982. West Antarctica: problem child of Gondwanaland. Tectonics 1, 3-19.

Elliot, D.H., 1996. The Hanson Formation: a new stratigraphical unit in the Transantarctic Mountains, Antarctica. Antarctic Science 8, 389-394.

Elliot, D.H., Fleming, T.H., 2004. Occurrence and dispersal of magmas in the Jurassic Ferrar Large Igneous Province, Antarctica. Gondwana Res. 7, 223-237.

Elliot, D.H., Fleming, T.H., in press. Physical volcanology and geological relationships of the Ferrar large Igneous Province, Antarctica. Journal of Volcanology and Geothermal Research.

Fanning, C.M., Moore, D.H., Bennett, V.C., Daly, S.J., 1996. The "Mawson Continent": Archaean to Proterozoic crust in East Antarctica and the Gawler Craton, Australia: A cornerstone in Rodinia and Gondwana. Geological Society of Australia, Abstracts 41, 135.

Fitzsimons, I.C.W., 2000. A review of tectonic events in the East Antarctic Shield and their implications for Gondwana and earlier supercontinents. Journal of African Earth Sciences 31, 3-23.

Fitzsimons, I.C.W., 2003. Proterozoic basement provinces of southern and southwestern Australia and their correlation with Antarctica. In: Yoshida, M., Windley, B.F. (Eds.), Proterozoic East Gondwana: Supercontinet assembly and breakup. Special Publication, vol. 206. Geological Society of London, pp. 93-130.

Ford, A.B., 1972. The Weddell Orogeny: latest Permian to earliest Mesozoic deformation at the Weddell Sea margin of the Transantarctic Mountains. In: Adie, R.J. (Ed.), Antarctic Geology and Geophysics. Universitetsforlaget, Oslo, pp. 419-425.

Geslin, J.K., Link, P.K., Fanning, C.M., 1999. High-precision provenance determination using detrital-zircon ages and petrography of Quaternary sands on the eastern Snake River Plain, Idaho. Geology 27, 295-298.

Goodge, J.W., Fanning, C.M., 1999. 2.5 b.y. of punctuated Earth history as recorded in a single rock. Geology 27, 1007-1010.

Goodge, J.W., Fanning, C.M., 2002. Precambrian history of the Nimrod Group, central Transantarctic Mountains. In: Gamble, J.A., Skinner, D.N.B., Henrys, S. (Eds.), Antarctica at the Close of a Millennium. Royal Society of New Zealand Bulletin, vol. 35, pp. 43-50.

Goodge, J.W., Fanning, C.M., Bennet, V.C., 2001. U-Pb evidence of $\sim 1.7 \mathrm{Ga}$ crustal tectonism during the Nimrod Orogeny in the Transantarctic Mountains, Antarctica: Implications for Proterozoic plate reconstructions. Precambrian Research 112, 261-288.

Goodge, J.W., Williams, I.S., Myrow, P., 2004a. Provenance of Neoproterozoic and lower Paleozoic siliciclastic rocks of the central Ross orogen, Antarctica: Detrital record of rift-, passive-, and active-margin sedimentation. Geological Society of America Bulletin 116, 1253-1279.

Goodge, J.W., Myrow, P., Philips, D., Fanning, C.M., Williams, I.S., 2004b. Siliciclastic record of rapid denudation in response to convergent-margin orogenesis, Ross orogen, Antarctica. In: Bernet, M., Spiegel, C. (Eds.), Detrital thermochronology. Special Paper, vol. 378. Geological Society of America, pp. 105-126.

Hallsworth, C.R., Morton, A.C., Claoué-Long, J., Fanning, C.M., 2000 Carboniferous sand provenance in the Pennine Basin, UK: constraints from heavy mineral and detrital zircon age data. Sedimentary Geology 137, 147-185.

Hälbich, I.W., 1992. The Cape Fold Belt orogeny: State of the art 1970s-1980s. In: De Wit, M.J., Ransome, G.D. (Eds.), Inversion tectonics of the Cape fold belt, Karoo and Cretaceous basins of Southern Africa: proceedings of the Conference on Inversion Tectonics of the Cape Fold Belt, Cape Town, South Africa. A.A. Balkema, Rotterdam, pp. 141-159.

Henjes-Kuntz, F., Kreuzer, H., 2003. Mid-Paleozoic igneous activity in northern Victoria Land, Antarctica: implications of new geochronological data. Geologisches Jahrbuch B 85, 271-302.

Isbell, J.L., 1991. Evidence for a low-gradient alluvial fan from the palaeoPacific margin in the upper Permian Buckley Formation, Beardmore Glacier area, Antarctica. In: Thomson, M.R.A., Crame, J.A., Thomson, J.W. (Eds.), Geological evolution of Antarctica. Cambridge University Press, Cambridge, pp. 215-217.

Isbell, J.L., 1999. The Kukri Erosion Surface; a reassessment of its relationship to the rocks of the Beacon Supergroup in the central Transantarctic Mountains, Antarctica. Antarctic Science 11, 228-238.
Isbell, J.L., Seegers, G.M., Gelhar, G.A., 1997. Upper Paleozoic glacial and post-glacial deposits, central Transantarctic Mountains, Antarctica. In: Martini, I.P. (Ed.), Late Glacial and Postglacial Environmental Changes: Quaternary, Carboniferous-Permian, and Proterozoic. Oxford University Press, Oxford, pp. 230-242.

Krull, E.S., Retallack, G.J., 2000. $\delta^{13} \mathrm{C}$ depth profiles from paleosols across the Permo-Triassic boundary: evidence for methane release. Geological Society of America Bulletin 112, 1459-1472.

Link, P.K., Fanning, C.M., Beranek, L.P., 2005. Reliability and longitudinal change of detrital-zircon age spectra in the Snake River system, Idaho and Wyoming: An example of reproducing the bumpy barcode. Sedimentary Geology 182, 101-142.

Ludwig, K.R., 2001. SQUID 1.02, A User's Manual; Berkeley Geochronology Center Special Publication. No. 2, 2455 Ridge Road, Berkeley, CA 94709, US.

Ludwig, K.R., 2003. Isoplot/Ex version 3.0: A geochronological toolkit for Microsoft Excel. Berkeley Geochronology Center Special Publication No. 4, 2455 Ridge Road, Berkeley CA 94709, USA.

Millar, I.L., Pankhurst, R.J., Fanning, C.M., 2002. Basement chronology of the Antarctic Peninsula: recurrent magmatism and anatexis in the Palaeozoic Gondwana margin. Journal of the Geological Society of London 159, $145-157$.

Mukasa, S.B., Dalziel, I.W.D., 2000. Marie Byrd Land, West Antarctica: Evolution of Gondwana's Pacific margin constrained by zircon $\mathrm{U}-\mathrm{Pb}$ geochronology and feldspar common-Pb isotopic compositions. Geological Society of America Bulletin 112, 611-627.

Mundil, R., Metcalfe, I., Ludwig, K.R., Renne, P.R., 2004. Age and timing of the Permian mass extinction: $\mathrm{U} / \mathrm{Pb}$ dating of closed-system zircons. Science 305 , $1760-1763$.

Paces, J.B., Miller, J.D., 1993. Precise U-Pb ages of Duluth Complex and related mafic intrusions, northeastern Minnesota: Geochronological insights to physical, petrogenetic, paleomagnetic, and tectonomagmatic processes associated with the 1.1 Ga Midcontinent Rift System. Journal of Geophysical Research 98 (13), 997-14013.

Pankhurst, R.J., Millar, I.L., Grunow, A.M., Storey, B.C., 1993. The preCenozoic magmatic history of the Thurston Island crustal block, West Antarctica. Journal of Geophysical Research 98, 11835-11850.

Pankhurst, R.J., Sorey, B.C., Millar, I.L., Macdonald, D.I.M., Vennum, W.R., 1988. Cambrian-Ordovician magmatism in the Thiel Mountains, Transantarctic Mountains, and implications for the Beardmore orogeny. Geology 16 , 246-249.

Pankhurst, R.J., Weaver, S.D., Bradshaw, J.D., Storey, B.C., Ireland, T.R., 1998. Geochronolgy and geochemistry of pre-Jurassic superterranes in Marie Byrd Land, Antarctica. Journal of Geophysical Research 103, 2529-2547.

Retallack, G.J., Seyedolali, A., Kroll, E.S., Hosier, W.T., Ambers, C.P., Kyte, F.T., 1998. Search for evidence of impact and the Permo-Triassic boundary in Antarctica and Australia. Geology 26, 979-982.

Ross, G.M., Villeneuve, M., 2003. Provenance of the Mesoproterozoic $(1.45 \mathrm{Ga})$ Belt Basin (western North America): Another piece in the preRodinia paleogeographic puzzle. Geological Society of America Bulletin 115, 1191-1217.

Ross, G.M., Parrish, R.R., Winston, D., 1992. Provenance and U-Pb geochronology of the Mesoproterozoic Belt Supergroup (northwestern United States): implications for age of deposition and pre-Panthalassa plate reconstructions. Earth and Planetary Science Letters 113, 57-76.

Rowell, A.J., van Schmus, W.R., Storey, B.C., Fetter, A.H., Evans, K.R., 2001. Latest Neoproterozoic to mid-Cambrian age for the main deformation phases of the Transantarctic Mountains: new stratigraphic and isotopic constraints from the Pensacola Mountains, Antarctica. Journal of the Geological Society of London 158, 295-308.

Sambridge, M.S., Compston, W., 1994. Mixture modeling of multi-component data sets with application to ion-probe zircon ages. Earth and Planetary Science Letters 128, 373-390.

Stump, E., 1995. The Ross orogen of the Transantarctic Mountains. Cambridge University Press, Cambridge. 284 pp.

Tera, F., Wasserburg, G.J., 1972. U-Th-Pb systematics in three Apollo 14 basalts and the problem of initial $\mathrm{Pb}$ in lunar rocks. Earth and Planetary Science Letters 14, 281-304. 
Vavra, C.L., Stanley, K.O., Collinson, J.W., 1981. Provenance and alteration of Triassic Fremouw Formation, central Transantarctic Mountains. In: Cresswell, M.M., Vella, P. (Eds.), Gondwana Five. A.A. Balkema, Amsterdam, pp. 149-153.

Vermeesch, P., 2004. How many grains are needed for a provenance study? Earth and Planetary Science Letters 224, 441-451.

Wandres, A.M., Bradshaw, J.G., 2005. New Zealand tectonostratigraphy and implications from conglomeratic rocks for the configuration of the SW pacific margin of Gondwana. In: Vaughan, A.P.M., Leat, P.T., Pankhurst, R.J. (Eds.), Terrane processes at the margins of Gondwana. Geol. Soc. Lond., Spec. Publ., vol. 246, pp. 179-216.

Wareham, C.D., Stump, E., Storey, B.C., Millar, I.L., Riley, T.R., 2001. Petrogenesis of the Cambrian Liv Group, a bimodal volcanic rock suite from the Ross orogen, Transantarctic Mountains, Antarctica. Geological Society of America Bulletin 113, 360-372.
Williams, I.S., 1998. U-Th-Pb geochronology by ion microprobe. In: McKibben, M.A., Shanks, W.C., Ridley, W.I. (Eds.), Applications of microanalytical techniques to understanding mineralizing processes. Reviews in Economic Geology, vol. 7, pp. 1-35.

Winberry, J.P., Anandakrishnan, S., 2004. The crustal structure of the West Antarctic rift system and Marie Byrd Land hotspot. Geology 32, 977-980.

Wysoczanski, R.J., Gibson, G.M., Ireland, T.R., 1997. SHRIMP dating of detrital zircons from the Takaka Terrane of New Zealand and Beacon supergroup, Antarctica: implications for source components and terrane accretion. In: Bradshaw, J.D., Weaver, S.D. (Eds.), Terrane Dynamics 97: International Conference on Terrane Geology; abstracts. University of Canterbury, Christchurch, pp. 170-172.

Wysoczanski, R.J., Forsyth, P.J., Woolfe, K.J., 2003. Zircon dating and provenance of rhyolite clasts in Beacon conglomerate, southern Victoria Land, Antarctica. Terra Antarctica 10, 567-580. 